\title{
On the generic existence of homoclinic points
}

\author{
FERNANDO OLIVEIRA \\ Departamento de Matemática, ICEX-UFMG, Catxa Postal 702, \\ 30161-Belo Horlzonte-MG, Brazll
}

(Recelved 17 January 1986 and revised 9 December 1986)

\begin{abstract}
This work is concerned with the generic existence of homoclinic points for area preserving diffeomorphisms of compact orientable surfaces We give a shorter proof of Pixton's theorem that shows that, $C^{r}$-generically, an area preserving diffeomorphism of the two sphere has the property that every hyperbolic periodic point has transverse homoclinic points Then, we extend Pixton's result to the torus and investigate certain generic aspects of the accumulation of the invariant manifolds all over themselves in the case of symplectic diffeomorphisms of compact manifolds
\end{abstract}

\section{Introduction and statement of results}

The concept of homoclinic points goes far back to Poincare who discovered the phenomenon in the 3-body problem [5] As realized by him and later investigated by Birkhoff [1], such points tremendously complicate the orbit structure of the system, for the behaviour of a diffeomorphism near the orbit of a transverse homoclinic point is statistical and can be described by a topological embedding of a subshift of finite type [8]

This work is concerned with the generic existence of homoclinic points in the case of area-preserving diffeomorphisms of surfaces

We consider a compact orientable surface $M$, a non-degenerate 2-form $\eta$ on $M$ and $D_{\eta}^{r}(M)$, the space of all $C^{r}$-diffeomorphisms $f$ of $M$ such that $f^{*} \eta=\eta$ with the $C^{r}$-topology, $1 \leq r \leq \infty$ The results are generic in the sense of Baire category If $p$ is a hyperbolic periodic point of $f \in D_{\eta}^{r}(M)$ with period $\tau$, then $p$ is a saddle, and the stable and unstable invariant manifolds of $p$ are

$$
W^{s}(p, f)=\left\{x \in M, f^{\tau n} x \rightarrow p, \quad \text { as } n \rightarrow \infty\right\}
$$

and

$$
W^{u}(p, f)=\left\{x \in M, f^{\tau n} x \rightarrow p, \quad \text { as } n \rightarrow-\infty\right\},
$$

respectively $W^{s}(p, f)$ and $W^{u}(p, f)$ are injectively immersed curves that intersect transversally at $p$ By Hartman's theorem, up to a contınuous change of coordinates, in a neighbourhood of $p, f^{\tau}$ looks like $(x, y) \rightarrow\left(\lambda^{-1} x, \lambda y\right)$, where $\lambda$ and $\lambda^{-1}$ are the elgenvalues of $d\left(f^{\tau}\right)_{p}$ and $0<|\lambda|<1$

A point $q$ in the intersection of $\bigcup_{j=0}^{\tau-1}\left[W^{s}\left(f^{\jmath} p, f\right) \backslash\left\{f^{\jmath} p\right\}\right]$ and $\bigcup_{j=0}^{\tau-1}\left[W^{u}\left(f^{j} p, f\right) \backslash\left\{f^{\jmath} p\right\}\right]$ is called homoclinic, and if the intersection is transversal, $q$ is a transverse homoclinic point 
Poincare suspected that transverse homoclinic points occur generically in the area-preserving case and conjectured that they would be dense in both invariant manifolds However, it was only in the early seventies that the first results appeared

In [10], Takens shows that for a generic $f$ in $D_{\eta}^{1}(M)$, if $p$ is a hyperbolic penodic point of $f$ then the transverse homochnic points of $p$ are dense in $W^{u}(p, f) \cup$ $W^{s}(p, f)$, but his methods are definitely restricted to the $C^{1}$-topology

In [11], Zehnder shows that, generically, in a very fine real analytic topology, every elliptic periodic point of a real analytic local diffeomorphism is a limit of transverse homoclinic points

In [4], Pixton shows that for a generic $f$ in $D_{\eta}^{r}\left(S^{2}\right)$, every hyperbolic periodic point of $f$ has transverse homoclinic points, but the argument there makes use of the Jordan curve theorem and therefore is restricted to the sphere $S^{2}$ Also, it is still not known whether or not the homoclinic points are $C^{r}$-generically dense in the union of the invariant manifolds for $r>1$, even in the case of the sphere

Here we give a different proof of Pixton's theorem and then extend it to the torus

A component of $W^{u}(p, f) \backslash\{p\}$ or $W^{s}(p, f) \backslash\{p\}$ is called a branch If $p$ has period $\tau$, then the period of its branches is $\tau$ when the eigenvalues of $d\left(f^{\tau}\right) p$ are positive, otherwise the period of the branches is $2 \tau$ We always order a branch so that $p$ is its infimum and use $L[a, b]$ and $L(a, b)$ to denote subintervals of $\{p\} \cup L$ from $a$ to $b$

If $E$ is a subset of $M$, we use $O_{k}^{\prime}(E, f)$ to denote $\bigcup_{k \leq \imath \leq \jmath} f^{x}(E),-\infty \leq k<\jmath \leq+\infty$, and $O_{+}(E, f)=O_{1}^{\infty}(E, f), O(E, f)=O_{-\infty}^{\infty}(E, f)$

For a compact subset $E$ of $M$, the $\omega$-limit set of $E$ with respect to $f$ is

$$
\omega(E, f)=\left\{x \in M, x=\lim _{i \rightarrow \infty} f^{n_{i}} x_{i}, n_{i} \rightarrow \infty, x_{i} \in E\right\}
$$

If $E$ is non-empty, then $\omega(E, f)$ is non-empty, compact, $f$-1nvariant and

$$
\overline{O_{k}^{\infty}(E, f)}=O_{k}^{\infty}(E, f) \cup \omega(E, f) \quad \text { for any } k
$$

We say that $x$ is $f$-recurrent if $x \in \omega(x, f)$

A branch of $f$ is a branch of some hyperbolic periodic point of $f$ If $L$ is an unstable branch of $f$ with period $\tau$ and $x \in L$, then $L=O\left(D, f^{\tau}\right)$, where $D=$ $L\left[x, f^{\tau} x\right]$ We define the $\omega$-limit set of $L$ with respect to $f$ as $\omega\left(D, f^{\tau}\right)$ and denote it by $\omega(L, f)$ or $\omega(L)$ The definition is independent of $D$, and $\bar{L}=L \cup \omega(L) \cup \inf L$ When $L$ is stable, we use $f^{-1}$ to define $\omega(L)$

In [2], when $r \geq 4$, Mather shows that for a generic $f$ in $D_{\eta}^{r}(M)$ any 2 branches of a hyperbolic periodic point of $f$ have the same closure

Our first result is the following

EVERYWHERE ACCUMULATION LEMMA Let fbe $a C^{1}$ area preserving diffeomorphism of a compact ortentable surface Assume that $L$ and $K$ are branches of $f$ with $L=K$ or $L \cap K=\varnothing$ If $K \cap \omega(L) \neq \varnothing$, then $K \subset \omega(L)$

On the other hand, it is not hard to show that for a $C^{r}$-generic area preserving diffeomorphism $f$, each unstable (stable) branch of $f$ has an $f$-recurrent $\left(f^{-1}\right.$. recurrent) point This together with the everywhere accumulation lemma implies the following genericity result, similar in flavour to that of Mather 
THEOREM 1 Let $M$ be a compact ortentable surface and $1 \leq r \leq \infty$ There is a residual subset $B$ of $D_{\eta}^{r}(M)$ such that any branch $L$ of $f \in B$ satisfies $L \subset \omega(L)$

The second result is the following

THEOREM 2 Let $f$ be a $C^{1}$ area-preserving diffeomorphism of the sphere or the torus, and $p$ a hyperbolic periodic point of $f$ If each unstable (stable) branch of $p$ has a $f$-recurrent ( $f^{-1}$-recurrent) point, then $p$ has homoclinic points

Theorem 2 implies Pixton's theorem, its extension to the torus, and we expect theorem 2 to be true in higher genus Its proof when $M$ is the torus involves lifting $f$ to an area-preserving diffeomorphism $F$ of the plane, looking at the accumulation of the $F$-branches of the fibre over $p$ over themselves, and 'closing the gates', an Idea introduced by Robinson [7], following earlier work by Newhouse

The author would like to thank Sheldon E Newhouse for the helpful conversations and CNPq for the support

\section{The everywhere accumulation lemma}

In this section we prove the everywhere accumulation lemma, which is a building block for most of the arguments in this work, and then derive theorem 1

LEMма 1 (Everywhere accumulation) Let $f$ be a $C^{1}$ area-preserving diffeomorphism of a compact orientable surface $M, K$ and $L$ branches of $f$ (not necessarily of the same orbut) such that $K \cap L=\varnothing$ or $K=L$ If $K \cap \omega(L) \neq \varnothing$, then $K \subset \omega(L)$

Proof Let $\tau$ be the least common multiple of the periods of $K$ and $L$ Since $L$ and $K$ are also branches of $f^{\tau}$, it suffices to consider the case when they are both fixed by $f$ Also, by replacing $f$ by $f^{-1}$ if necessary, we assume that $K$ is unstable

We prove only the case when $L$ is unstable, since a slight modification of the argument works when $L$ is stable

Let $V$ be a neighbourhood of inf $K$ where $f$ is almost linear Since $\omega(L)$ and $K$ are $f$-invariant, $\omega(L) \cap K$ has a point $x$ inside $V$ By the same reason, it is enough to show that $K\left(f^{-1} x, x\right) \subset \omega(L)$, and for this, it suffices to prove that for any $z \in K\left(f^{-1} x, x\right)$ and $\varepsilon>0$, we have

$$
B_{\varepsilon}\left(f^{-t} z\right) \cap L \neq \varnothing, \quad \text { for some } \imath \in\{0, \quad, 2 g\},
$$

where $g$ is the genus of $M$

Let $n$ be an integer satisfying

$$
\mu(M)<n \min \left\{\mu\left(B_{\varepsilon}\left(f^{-1} z\right)^{+}\right), \mu\left(B_{\varepsilon}\left(f^{-\imath} z\right)^{-}\right), 0 \leq \imath \leq 2 g\right\},
$$

where $\mu$ is the measure induced by $\eta$ and $B_{\varepsilon}\left(f^{-i} z\right)^{+}$and $B_{\varepsilon}\left(f^{-t} z\right)^{-}$are the upper and lower components of $B_{\varepsilon}\left(f^{-i} z\right) \backslash K[\operatorname{lnf} K, x]$, respectively

Let $B$ be a closed ball centred at $x$ and small enough that $B, \quad, f^{-(n+2 g)} B$ are pairwise disjoint, contained in $V$ and disjoint from $\bigcup_{t=0}^{2 g} B_{\varepsilon}\left(f^{-t} z\right)$ Let $q=$ $\inf \left\{p \in L \backslash K^{\prime}, p \in B\right\}$, where $K^{\prime}$ is the connected component of $K \cap V$ containing inf $K$, that is, $q$ is the first point of $L \backslash K^{\prime}$ to intersect $B$, and assume for definiteness that $q$ is above $K$ inside $V$ Join $q$ and $x$ by a simple curve $\gamma$ contained in $B$, and 
consider the set

$$
\Gamma=O_{-(2 \mathrm{~g}+1)}^{0}(\gamma) \cup K\left[f^{-(2 g+1)} x, x\right]
$$

For $0 \leq t \leq 2 g$, let $q_{t}=\sup \left\{p \in L\left[f^{-(1+1)} q, f^{-1} q\right), p \in \Gamma\right\}$, that is, $q_{t}$ is the first point where $L$ intersects $\Gamma$ as we move along $L$ from $f^{-t} q$ to $f^{-(t+1)} q$ If $l_{i}=L\left[q_{1}, f^{-t} q\right]$, then $\{l, 0 \leq \imath \leq 2 g\}$ is a collection of $2 g+1$ simple curves that intersect one another and $\Gamma$ at most at their endpoints $q$, and $f^{-1} q, 0 \leq t \leq 2 g$ (See figure 21 )

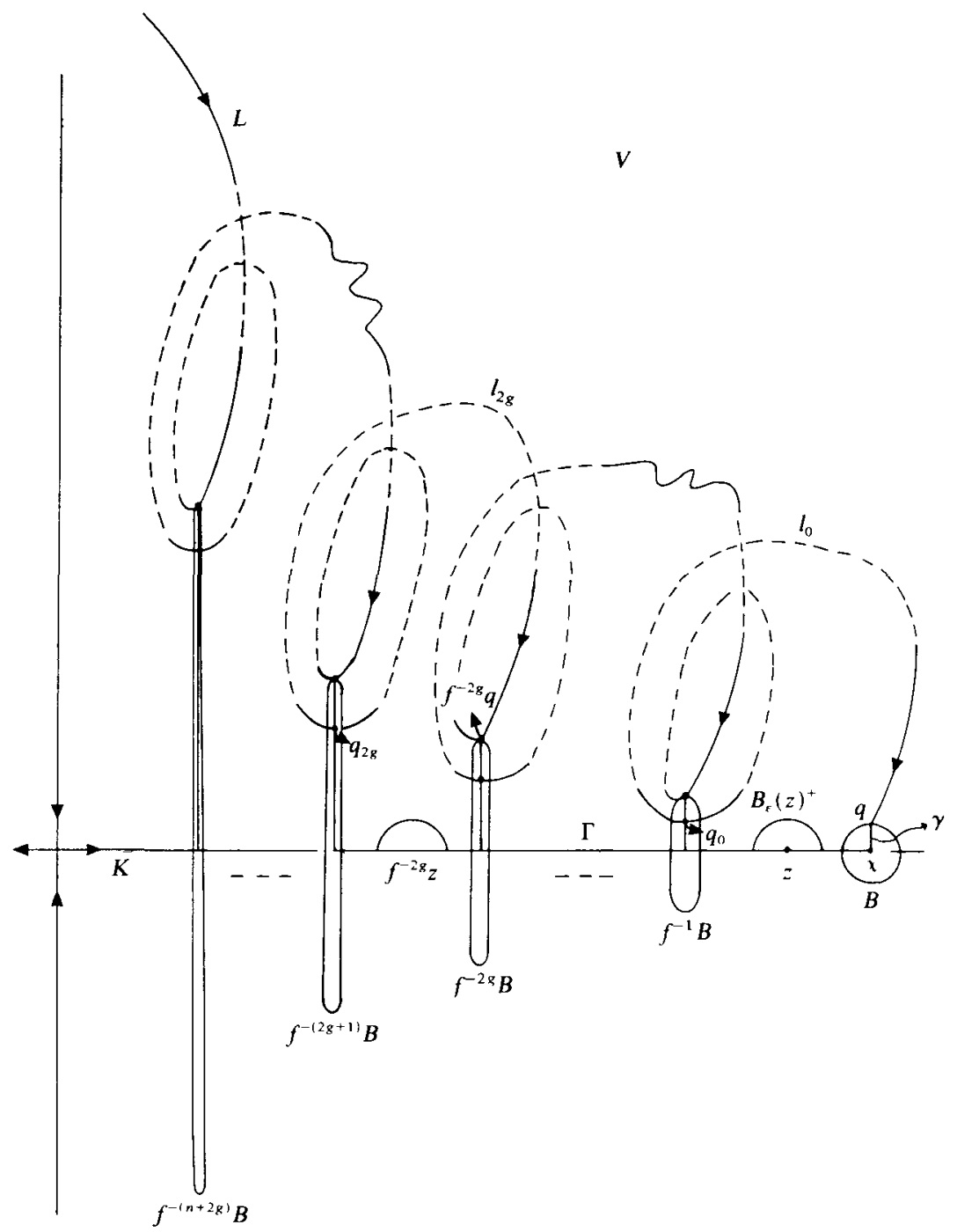

FIGURE 21

Now, if we collapse $\Gamma$ into a point, then the resulting set with the quotient topology is still a compact orientable surface of genus $g$ and $\{l, 0 \leq \imath \leq 2 g\}$ becomes a collection of $2 g+1$ simple closed curves that interect exactly at one point, namely, 
the point obtained by collapsing $\Gamma$ Since in a compact orientable surface of genus $g$, any collection of $2 g+1$ simple closed curves that interect exactly at one point disconnects the surface, the set $M^{*}=M \backslash\left(\Gamma \cup l_{0} \cup \quad \cup I_{2 g}\right)$ is disconnected

We claim that the boundary of any component $A$ of $M^{*}$ intersects $K\left[f^{-(2 g+1)} x, x\right]$ To prove this, we need the following facts, obvious from the above construction

(1) $\partial\left(M^{*}\right)=K\left[f^{-(2 g+1)} x, x\right] \cup O_{-(2 g+1)}^{0}(\gamma) \cup l_{0} \cup \cup l_{2 g}$

(2) If $\partial A \cap\left(l_{j} \backslash\left\{q_{j}\right\}\right) \neq \varnothing$, then $l_{j} \subset \partial A$ and $f^{-J} q \in \partial A \cap f^{-J} \gamma$

(3) If $q_{j} \in f^{-1} \gamma$, then $j<t$

By (1),

$$
\partial A \subset K\left[f^{-(2 g+1)} x, x\right] \cup O_{-(2 g+1)}^{0}(\gamma) \cup l_{0} \cup \cup l_{2 g},
$$

and by (2),

$$
\partial A \cap\left(K\left[f^{-(2 g+1)} x, x\right] \cup O_{-(2 g+1)}^{0}(\gamma)\right) \neq \varnothing
$$

If $\partial A \cap K\left[f^{-(2 g+1)} x, x\right] \neq \varnothing$, we are done So assume that $\partial A \cap O_{-(2 g+1)}^{0}(\gamma) \neq \varnothing \mathrm{We}$ have that the set

$$
I=\left\{k \in\{0, \quad, 2 g+1\}, \partial A \cap f^{-k} \gamma \neq \varnothing\right\}
$$

15 not empty Let $t=\inf I$

It follows that $f^{-t} \gamma \subset \partial A$ In fact, if this were false, then for some $k,\left(l_{k} \backslash\left\{q_{k}\right\}\right) \cap \partial A \neq$ $\varnothing$, (see figure $22(\mathrm{a})$ )

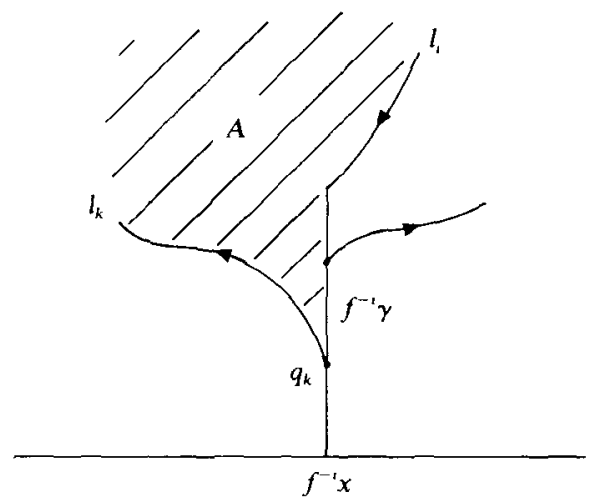

FIGURE $22(a)$

By (2) $f^{-k} \gamma \cap \partial A \neq \varnothing$, and by (3) we have $k<\imath$, contradictıng the choice of 1 This proves the claim

Since $K \cap L=\varnothing$ or $K=L$, we may choose a thin rectangular open strip $S$ based over $K(x, f x)$ with sides in $\gamma$ and $f \gamma$, and such that $O_{-(n+2 g)}^{0}(S) \cap L[\operatorname{1nf} L, q]=\varnothing$ It follows from the claim that each component of $M^{*}$ contains $f^{-i} S$ for some $t \in\{0, \quad, 2 g+1\}$ Take $d \in\{1, \quad, 2 g+1\}$ minimal such that $f^{-d} S$ and $f^{-(d-1)} S$ are not in the same component of $M^{*}$, and let $C$ be the component of $M^{*}$ containing $f^{-d} S$, (see figure $22(\mathrm{~b})$ ) 


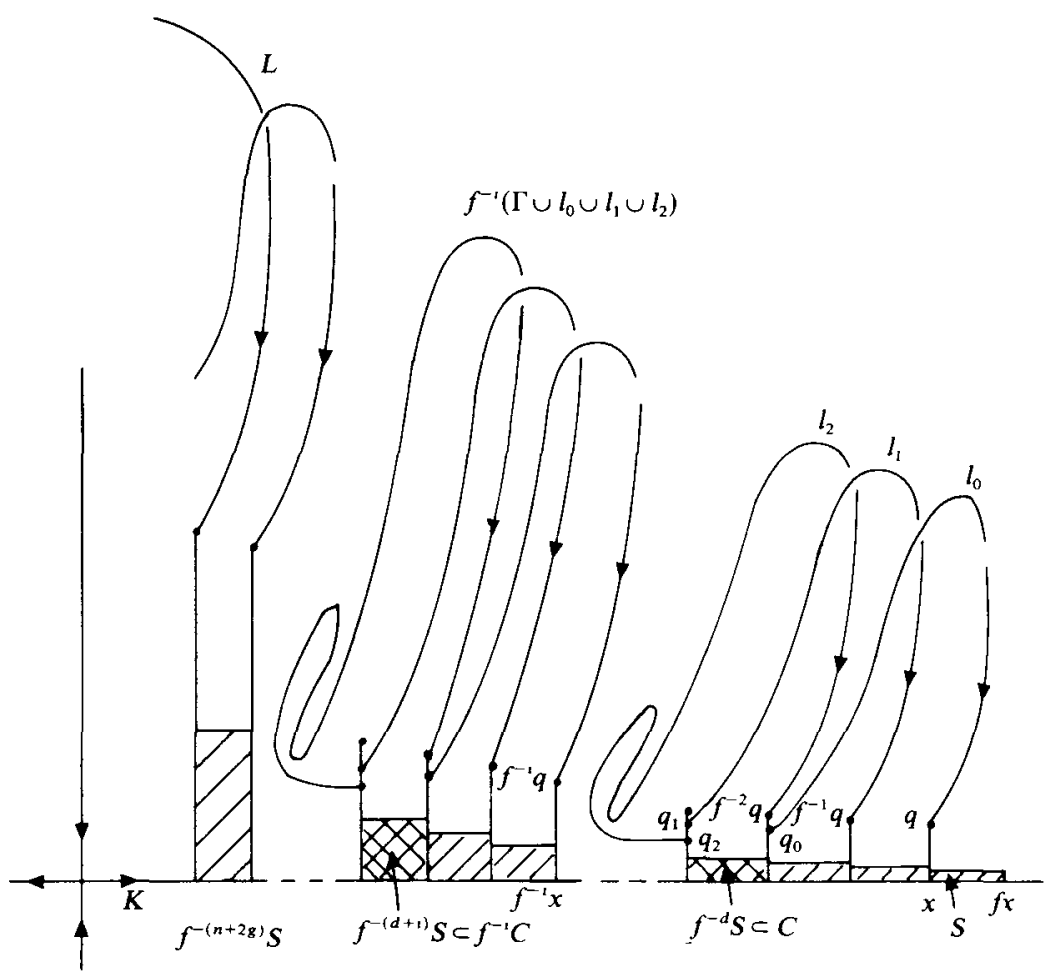

FIGURE $22(b) g=1, d=3$

Then $f^{-d} S \subset C$ and $S, \quad, f^{-(d-1)} S$ all belong to a single component of $M^{*}$ different from $C$, implyıng that for $0 \leq \imath \leq n-1, f^{-1} C$ is the component of $f^{-1}\left(M^{*}\right)$ containing $f^{-(t+d)} S$, and $f^{-1} S, \quad, f^{-(i+d-1)} S$ all belong to a single component of $f^{-i}\left(M^{*}\right)$ different from $f^{-i} C$

On the other hand, $\partial\left(M^{*}\right) \subset \Gamma \cup L\left[f^{-(2 g+1)} q, q\right]$, and for $0 \leq \imath \leq n-1$, we have

$$
\partial\left(f^{-\imath} M^{*}\right)=f^{-\imath}\left(\partial M^{*}\right) \subset f^{-1} \Gamma \cup L\left[f^{-(\imath+2 g+1)} q, f^{-t} q\right],
$$

implying that $S, \quad, f^{-i} S$ all belong to a single component of $f^{-1} M^{*}$

It follows that for $0 \leq \imath \leq n-1, S, \quad, f^{-(1+d-1)} S$ are all in a single component of $f^{-\imath} M^{*}$ different from $f^{-\imath} C$ In particular, if $\imath \neq 0$, then $f^{-d} S \cap f^{-\imath} C \neq \varnothing$, and $D=$ $C \backslash O_{-(n-1)}^{-1}(C) \neq \varnothing$, since $f^{-d} S \subset D$

Now, $D, f^{-1} D, \quad, f^{-(n-1)} D$ are pairwise disjoint and therefore

$$
\mu(D) \leq \frac{1}{n} \mu(M)<\mu\left(B_{\varepsilon}\left(f^{-d+1} z\right)^{+}\right)
$$

1mplying that $B_{\varepsilon}\left(f^{-d+1} z\right)^{+} \not \subset D$, or $B_{\varepsilon}\left(f^{-d+1} z\right)^{+} \cap(M \backslash D) \neq \varnothing$ (See figure 23$)$ On the other hand, since $f^{-d} S \subset D$, we have that $B_{\varepsilon}\left(f^{-d+1} z\right)^{+} \cap D \neq \varnothing$ and therefore $B_{\varepsilon}\left(f^{-d+1} z\right)^{+} \cap \partial D \neq \varnothing$ 


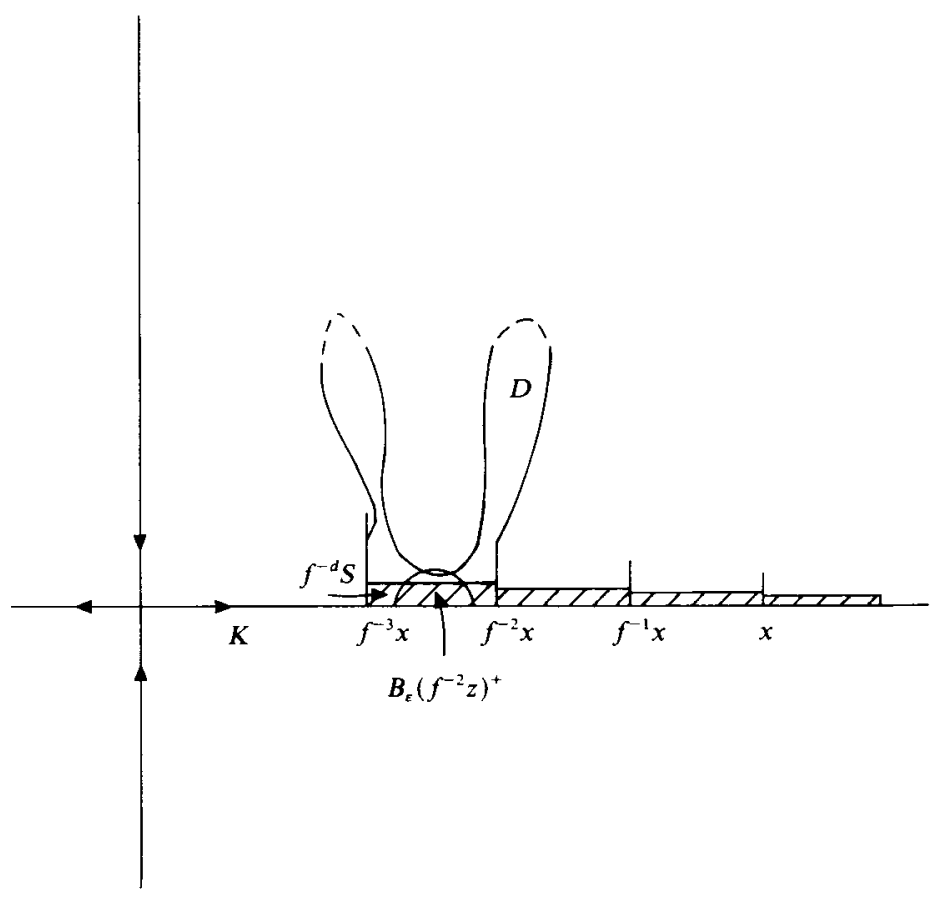

FIGURE $23 g=1, d=3$

But

$$
\begin{aligned}
\partial D & \subset O_{-(n-1)}^{0}(\partial C) \subset O_{-(n-1)}^{0}\left(\partial M^{*}\right) \\
& \subset O_{-(n-1)}^{0}\left(\Gamma \cup L\left[f^{-(2 g+1)} q, q\right]\right) \\
& \subset K[\inf K, x] \cup L[\inf L, q] \cup O_{-(n+2 g)}^{0}(\gamma),
\end{aligned}
$$

and by the choice of $\gamma$, we have that

$$
B_{\varepsilon}\left(f^{-d+1} z\right)^{+} \cap\left[K[\operatorname{lnf} K, x] \cup O_{-(n+2 g)}^{0}(\gamma)\right]=\varnothing
$$

It follows that $L[\inf L, q] \cap B_{\varepsilon}\left(f^{-d+1} z\right)^{+} \neq \varnothing$

We would like to give an example to show that lemma 1 is false if the area-preserving assumption is dropped

Consider the unit sphere and the gradient flow of $F(x, y, z)=z^{2}-y^{2}$, whose set of critical elements consists of two saddles $S_{1}=(1,0,0)$ and $S_{2}=(-1,0,0)$, two sources and two sinks, as shown in figures $24(\mathrm{a})$ and $24(\mathrm{~b})$ Let $f$ be its time-one map and consider the open sets $A$ and $B$ shown in figure 24(c) Isotopically change $f$ inside $A$ and $B$, in order to create heteroclinic points inside $A$ and a heteroclinic tangency inside $B$, as shown in figure 24(c) If $K$ is the perturbed unstable branch of $S_{1}$, then $K$ accumulates all over the unstable manifold of $S_{2}$, but on exactly one orbit of the stable branch $L$, as shown in figure $24(\mathrm{~d})$ 


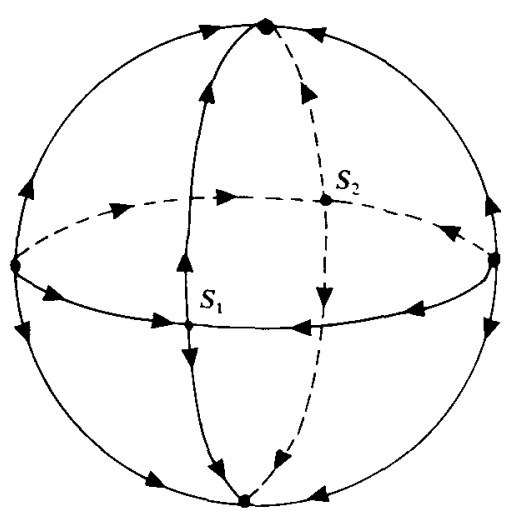

(a)

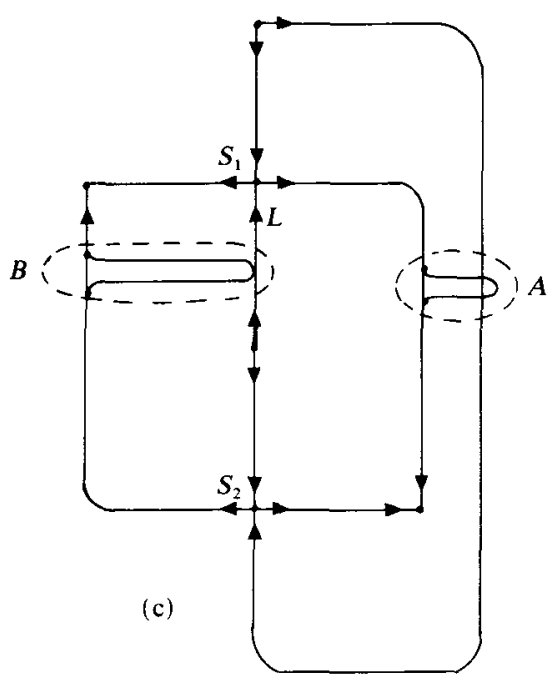

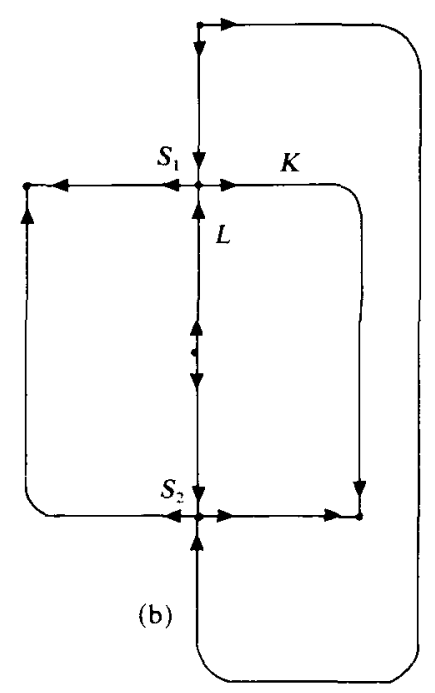

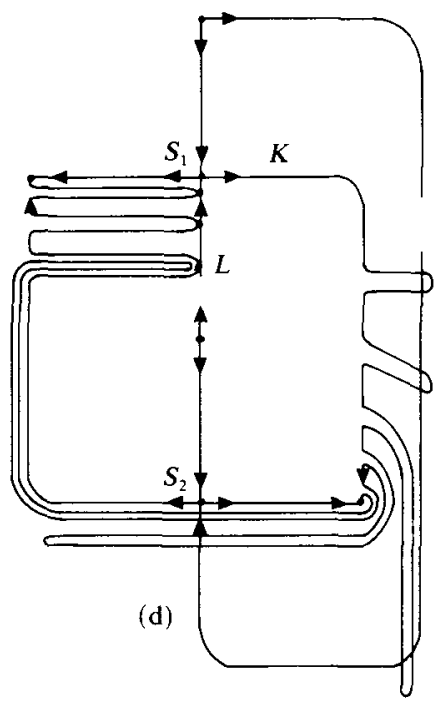

Figure 24

The next lemma is a variation of lemma 1 that will be needed in $\S 3$ when we work with the universal cover of $M$

LEMMA 2 Let $\hat{M}$ be an open simply connected surface, $\eta$ a non-degenerate 2-form on $\hat{M}$ and $f$ a $C^{1}$ diffeomorphism of $\hat{M}$ such that $f^{*} \eta=\eta$

Let $K$ and $L$ be branches of $f$ and assume that $L$ and inf $K$ are contained in a simply connected open subset $A$ of $\hat{M}$ satisfying $\mu(A)<+\infty$, where $\mu$ is the measure induced by $\eta$

If $K \cap L=\varnothing$ or $K=L$, then $K \cap \omega(L) \neq \varnothing$ implies that $K \subset \omega(L)$ 
Proof We may assume that $L$ and $K$ are unstable and fixed by $f$

Let $V \subset A$ be a neighbourhood of inf $K$ where $f$ is almost linear and take $x \in \omega(L) \cap K \cap V$ It suffices to show that for any $\varepsilon>0$ and $z \in K\left(f^{-1} x, x\right)$, we have $B_{\varepsilon}(z) \cap L \neq \varnothing$

Let $n$ be an integer satısfying

$$
\mu(A)<n \min \left\{\mu\left(B_{\varepsilon}(z)^{+}\right), \mu\left(B_{\varepsilon}(z)^{-}\right)\right\}
$$

and $B$ a closed ball centred at $x$ and small enough so that $B, \quad, f^{-n} B$ are pairwise disjoint, contained in $V$ and disjoint from $B_{\varepsilon}(z)$

Let $q=\inf \{p \in L, p \in B\}$ and assume for definiteness that $q$ is above $K$ inside $V$ Join $q$ and $x$ by a simple curve $\gamma$ contained in $B$ and let $S$ be a thin rectangular open strip based over $K\left(f^{-1} x, x\right)$ such that

$$
O_{-(n-1)}^{0}(S) \cap L[\inf L, f(q)]=\varnothing
$$

We claim that there is an open set $C$ homeomorphic to a disk with the following properties

(1) $\partial C \subset K\left[f^{-1} x, x\right] \cup \gamma \cup f^{-1} \gamma \cup L[\operatorname{lnf} L, f(q)]$

(2) $S \subset C$ and $S \cap f^{-\imath} C=\varnothing$ for $1 \leq \imath \leq n-1$

(3) $f^{-i}(C) \subset A$ for $0 \leq t \leq n-1$

Assuming the claim, the lemma is proved as follows

Let $D=C \backslash\left(f^{-1} C \cup \cup f^{-(n-1)} C\right)$ By (2), $S \subset D$ Also, $D, \quad, f^{-(n-1)} D$ are pairwise disjoint, and by (3) they are all contained in $A$ Therefore $\mu(D)<$ $(1 / n) \mu(A)<\mu\left(B_{\varepsilon}(z)^{+}\right)$1mplying that $B_{\varepsilon}(z)^{+} \not \subset D$ or $B_{\varepsilon}(z)^{+} \cap(\hat{M} \backslash D) \neq \varnothing$ On the other hand, since $S \subset D$, we have that $B_{\varepsilon}(z)^{+} \cap D \neq \varnothing$, and therefore $B_{\varepsilon}(z)^{+} \cap \partial D \neq$ $\varnothing$ Now, by the definition of $D$ and (1), we have that

$$
\partial D \subset O_{-(n-1)}^{0}(\partial C) \subset K\left[f^{-n} x, x\right] \cup O_{-n}^{0}(\gamma) \cup L[\operatorname{lnf} L, f(q)],
$$

and since $B_{\varepsilon}(z)^{+} \cap\left(K\left[f^{-n} x, x\right] \cup O_{-n}^{0}(B)\right)=\varnothing \quad$ it follows that $B_{\varepsilon}(z)^{+} \cap$ $L[\inf L, f(q)] \neq \varnothing$

Now it remains to prove the claim

Let $q_{0}=\sup \left\{p \in L\left[f^{-1} q, q\right], p \in f^{-1} \gamma\right\}$ and $\gamma_{0}$ be the subınterval of $f^{-1} \gamma$ from $f^{-1} x$ to $q_{0}$ If

$$
\alpha=\gamma_{0} \cup L\left[q_{0}, q\right] \cup K\left[f^{-1} x, x\right]
$$

then $\alpha$ is a simple closed curve Let $E$ be the component of $\hat{M} \backslash \alpha$ homeomorphic to an open disk Since $S \cap \alpha=\varnothing$, we have that $S$ is contained in one of the components of $\hat{M} \backslash \alpha$, and that $S$ and $K\left[\operatorname{lnf} K, f^{-1} x\right]$ are in different components of $\hat{M} \backslash \alpha$

Firstly we consider the case when $S \subset E$ Take $C=E$ Since $\alpha=\partial C, C$ satisfies (1) New, for $1 \leq \imath \leq n-1$ we have

$$
\begin{aligned}
\partial\left(f^{-1} C\right) & =f^{-1}(\partial C) \\
& =f^{-1} \alpha \\
& \subset f^{-(1+1)} \gamma \cup f^{-1} \gamma \cup K\left[f^{-(t+1)} x, f^{-1} x\right] \cup L\left[\operatorname{1nf} L, f^{-\imath} q\right]
\end{aligned}
$$

and therefore $f^{-1} S$ and $S$ are in different components of $\hat{M} \backslash \partial\left(f^{-t} C\right)$, implying that $C$ satisfies (2) Finally, $C$ satisfies (3) since $A$ is simply connected and $\partial\left(f^{-1} C\right)=$ $f^{-\imath} \alpha \subset A$ for $0 \leq \imath \leq n-1$ 
Now let us consider the case when $K\left[\operatorname{lnf} K, f^{-1} x\right) \subset E$ Firstly we show that $L\left(q_{0}, q\right) \cap f^{-1} \gamma_{0} \neq \varnothing$ If this is false then $f^{-1} \gamma_{0} \cap \alpha=\varnothing$ and therefore $f^{-1} q_{0} \in E$ (see figure 2 5) Since $L\left[f^{-1} q_{0}, f^{-1} q\right) \cap \alpha=\varnothing$, we have that $L\left[f^{-1} q_{0}, f^{-1} q\right) \subset E$, implying that $f^{-1} \alpha \cap \alpha=\gamma_{0}$ and $f^{-1} \alpha \subset \bar{E}$ It follows that the component of $\hat{M} \backslash f^{-1} \alpha$ homeomorphic to an open disk contains $f^{-1} S$, implying that $S \subset E$, a contradiction

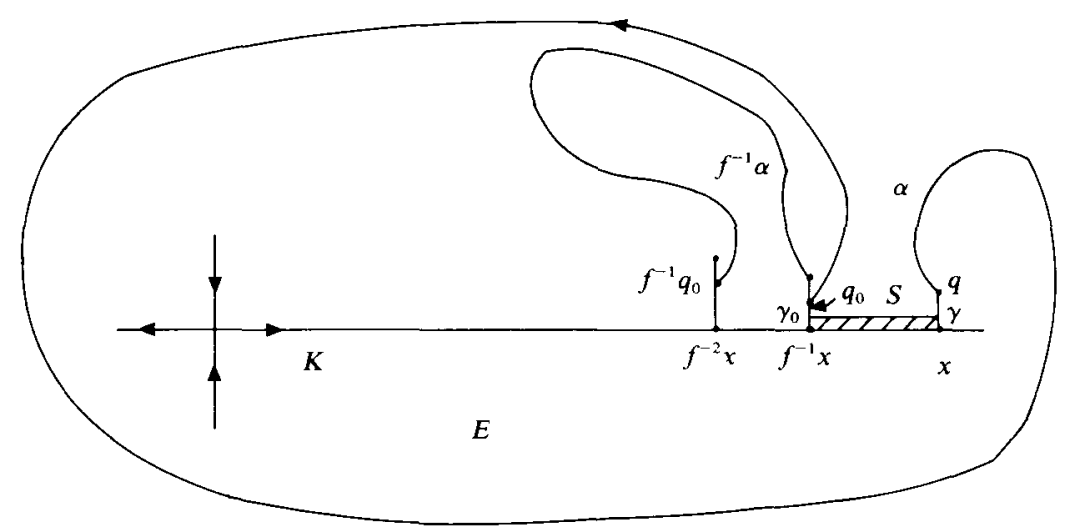

FIGURE 25

Now that $L\left(q_{0}^{\prime}, q\right) \cap f^{-1} \gamma_{0} \neq \varnothing$, order $f^{-1} \gamma_{0}$ so that $f^{-2} x=\inf f^{-1} \gamma_{0}$, and let $q_{1}=\operatorname{lnf}\left\{p \in f^{-1} \gamma_{0} / p \in L\left(q_{0}, q\right)\right\}$ It follows that $K\left[f^{-2} x, f^{-1} x\right] \cup f^{-1} \gamma_{0}\left[f^{-2} x, q_{1}\right]$ is a simple curve contained in $E$, except for its endpoints $f^{-1} x$ and $q_{1}$, that belong to $\partial E=\alpha$ (See figure 26 ) If we define

$$
\alpha_{1}=K\left[f^{-2} x, f^{-1} x\right] \cup f^{-1} \gamma_{0}\left[f^{-2} x, q_{1}\right] \cup L\left[q_{0}, q_{1}\right] \cup \gamma_{0},
$$

then $\alpha_{1}$ is a simple closed curve, and if $F$ is the component of $\hat{M} \backslash \alpha_{1}$ homeomorphic to an open disk, then $f^{-1} S \subset F$

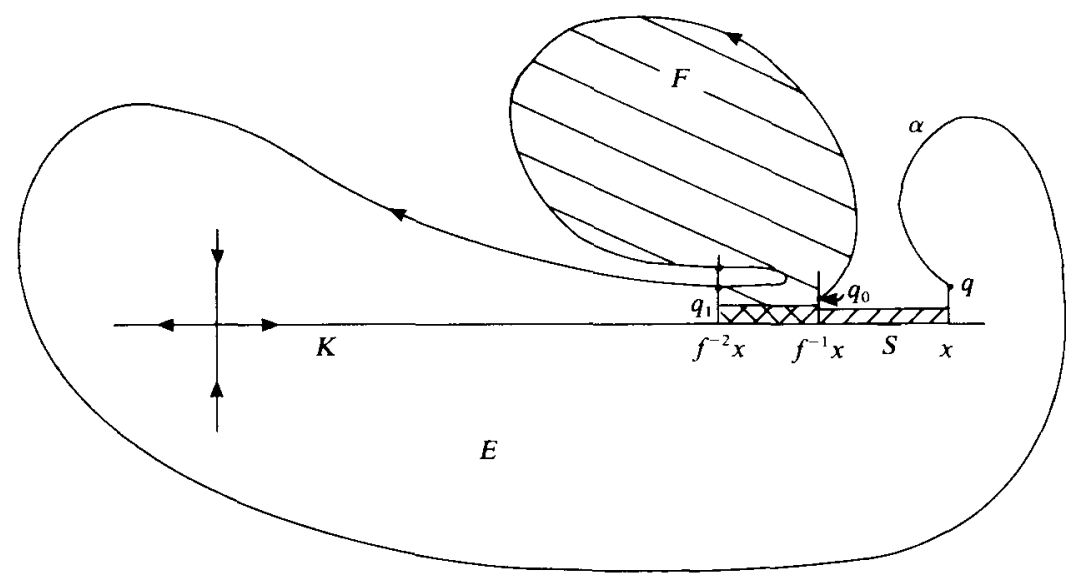

FIGURE 26 
Let $C=f(F)$

$$
\partial C=f\left(\alpha_{1}\right)=K\left[f^{-1} x, x\right] \cup \gamma_{0}\left[f^{-1} x, f\left(q_{1}\right)\right] \cup L\left[f\left(q_{0}\right), f\left(q_{1}\right)\right] \cup f\left(\gamma_{0}\right)
$$

implies that

$$
\partial C \subset K\left[f^{-1} x, x\right] \cup f^{-1} \gamma \cup L[\operatorname{lnf} L, f(q)] \cup \gamma,
$$

that is, $C$ satisfies (1) Now, $f^{-1} S \subset F$ implies $S \subset C$, and since for $1 \leq 1 \leq n-1, S$ and $f^{-i} S$ are in different components of $\hat{M} \backslash \partial\left(f^{-1} C\right)$, we have that $S \cap f^{-i} C=\varnothing$ for $1 \leq \imath \leq n-1$, and $C$ satisfies (2) (3) follows from the fact that $A$ is simply connected and $\partial C=f\left(\alpha_{1}\right) \subset A$

Now we head towards the proof of theorem 1 Four more lemmas are necessary

LEMMA 3 For $1 \leq r \leq \infty, D_{\eta}^{r}(M)$ contains a residual subset $B$ such that if $L$ is an unstable (stable) branch of $f \in B$, then $L$ has a f-recurrent $\left(f^{-1}\right.$-recurrent) point

Proof We take a Riemannian metric on $M$ and denote by length $(\alpha)$ the length of a curve $\alpha$

If $L$ is an unstable (stable) branch of $f$ and length $(L)>1 / k$, we use $D_{k}(L)$ to denote the fundamental interval $L\left[q, f^{\tau} q\right],\left(L\left[f^{\tau} q, q\right]\right)$, where $\tau$ is the period of $L$ and $q=q_{k}(i)$ is the point of $L$ for which length $(L[\operatorname{lnf} L, q])=1 / k$.

Let $A_{k, m}^{n}$ be the set of $f \in D_{\eta}^{r}(M)$ with the property that all periodic points of period $\leq n$ are elementary and any branch $L$ of $f$ satisfying

(1) period of inf $L \leq n$, and

(2) length $(L)>1 / k$,

also satisfies

(3) $d\left[O_{+}\left(q_{k}(L), f\right), q_{k}(L)\right]<1 / m$, when $L$ is unstable, or $d\left[O_{+}\left(q_{k}(L), f^{-1}\right)\right.$, $\left.q_{k}(L)\right]<1 / m$, when $L$ is stable

Since conditions (1), (2) and (3) are open and the set of hyperbolic periodic points of $f \in A_{k, m}^{n}$ with period $\leq n$ varies contınuously with $f, A_{k, m}^{n}$ is open for all $n, k, m \in \mathbb{N}$

Now we want to show that $A_{k, m}^{n}$ is dense in $D_{\eta}^{r}(M)$ Let $U$ be an open subset of $D_{\eta}^{r}(M)$ In [6], Robınson shows that for generic $f \in D_{\eta}^{r}(M)$, every periodic point of $f$ is elementary Let $f \in U$ have this property

If no branch of $f$ satisfies (1) and (2), then $f \in A_{k, m}^{n}$ So, assume that $f$ has at least one branch $L$ that satisfies (1) and (2) If $s$ is the number of hyperbolic periodic points of $f$ with period $\leq n$, then $f$ has at most $4 s$ of such branches The idea is to make small changes in $f$ and obtain another diffeomorphism $g \in U$ with the property that any branch of $g$ that satisfies (1) and (2) also satisfies (3)

For this, we need the following perturbation lemma

LEMMA 4 Let $\varepsilon>0$ and $\rho>0$ Then there is $\delta>0$ such that for any $p \in B_{\delta}(0)$, there is a $C^{\infty}$ area-preserving diffeomorphism $\Phi \mathbb{R}^{2} \rightarrow \mathbb{R}^{2}$ such that

(1) $\Phi(0)=p$,

(2) $\Phi=1 \mathrm{~d}$ off of $B_{\rho}(0)$,

(3) $\|\Phi-1 \mathrm{~d}\|_{r}<\varepsilon$,

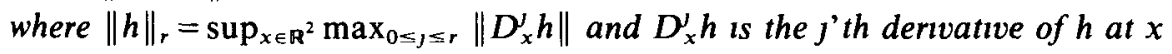


The proof of this lemma involves the use of generating functions (see [3]) Roughly speaking, the idea is the following

Take $\sigma \in(0, \rho)$ and a bump function $\alpha \mathbb{R}^{2} \rightarrow \mathbb{R}$ with $\alpha=1$ on $B_{\sigma}(0)$ and $\alpha=0$ on $\mathbb{R}^{2} \backslash B_{\rho}(0)$

Let $S(x, z)=x z+(a z-b x) \quad \alpha(x, z)$, for $p=(a, b)$ in $B_{\sigma}(0)$, and consider

$$
\begin{aligned}
& f(x, z)=\left(x, S_{x}(x, z)\right) \\
& g(x, z)=\left(S_{z}(x, z), z\right)
\end{aligned}
$$

If $\|p\|$ is small enough, $f$ and $g$ are diffeomorphisms, and $\Phi=g \circ f^{-1}$ is the desired area preserving diffeomorphism

Returning to the proof of lemma 3 , let $V$ be a neighbourhood of $f$ such that $V \subset U$ and every $h \in V$ also has $s$ hyperbolic periodic points of period $\leq n$

Let $L$ be an unstable branch of $f$ that satisfies (1) and (2) and $W$ a neighbourhood of $f^{\tau}\left(q_{k}(L)\right)$ such that

(a) $W \cap O_{-\infty}^{-1}\left(q_{k}(L), f\right)=\varnothing$,

(b) $W, f^{-1} W, \quad f^{-\tau} W$ are pairwise disjoint,

(c) $f^{-\tau} W \subset B_{1 / m}\left(q_{k}(L)\right)$,

where $B_{\delta}(q)$ denotes the open neighbourhood of radius $\delta$ around $q$

Assume that $L$ is unstable

Almost every point of $W$ returns to $W$ in the future Let $y$ be a recurrent point of $W$ sufficiently close to $f^{\tau} q_{k}(L)$ Lemma 4 (with the and of a symplectic chart) gives a diffeomorphism $\phi \in D_{\eta}^{r}(M)$ supported on $W$, as $C^{r}$-close as we want to the identity, and such that $\phi\left(f^{\tau} q_{k}(L)\right)=y$

Consider $f_{1}=\phi \circ f$ We can have $f_{1}$ as $C^{r}$-close as we want to $f$ by choosing $y$ close enough to $f^{\tau} q_{k}(L)$ Since $f^{-1}=f_{1}^{-1}$ off of $W$, by (a) we have that $\inf L$ is a hyperbolic periodic point of $f_{1}$, and more precisely, inf $L$ is the infimum of the perturbed branch $L_{1}=O\left(\phi\left(D_{k}(L)\right), f_{1}^{\tau}\right)$ which contains $y$, since $\phi\left(f^{\tau} q_{k}(L)\right)=y$ Note that by the same reason we have $f^{-J} D_{k}(L)=f_{1}^{-J} \phi\left(D_{k}(L)\right)$ for all $J \geq 1$, and therefore $D_{k}\left(L_{1}\right)=\phi\left(D_{k}(L)\right)$ and $q_{k}(L)=q_{k}\left(L_{1}\right)$

Let $n=\operatorname{nnf}\left\{y \geq 1 / f^{y} y \in W\right\}$ Since $f=f_{1}$ off of $f^{-1} W$, the positive orbit of $y$ under $f$ and $f_{1}$ coincide up to $n-1$ iterates, and both $f_{1}^{n}(y)=\phi\left(f^{n}(y)\right)$ and $f^{n}(y)$ belong to $W$ By (b), $n>\tau$ and $f_{1}^{n-t}(y)=f^{n-t}(y) \in f^{-1} W$, for $1 \leq \imath \leq \tau$ (c) implies that $f_{1}^{n-\tau}(y) \in B_{1 / m}\left(q_{k}(L)\right)$, and since $f_{1}^{-\tau}(y)=q_{k}(L)=q_{k}\left(L_{1}\right)$, we have $f_{1}^{n}\left(q_{k}\left(L_{1}\right)\right) \in$ $B_{1 / m}\left(q_{k}\left(L_{1}\right)\right)$, showing that $d\left[O_{+}\left(q_{k}\left(L_{1}\right), f_{1}\right), q_{k}\left(L_{1}\right)\right]<1 / m$

Summarizing, by an arbitrarily small change in the $C^{r}$-topology, we change from $f$ to $f_{1} \in V \subset U$ so that a branch of $L$ that satisfies (1) and (2) is perturbed into a branch $L_{1}$ of $f_{1}$ that satisfies the condition in the definition of $A_{k m}^{n}$

If $M_{1} \neq L_{1}$ is a branch of $f_{1}$ that satisfies (1) and (2), then we repeat the above construction and change from $f_{1}$ to $f_{2} \in V \subset U$, so that $M_{1}$ is perturbed into a branch $M_{2}$ of $f_{2}$ that satisfies the condition in the definition of $A_{k, m}^{n}$ Since this is an open condition, if our second change is small enough, $L_{1}$ changes to a branch $L_{2}$ (or it does not change at all) which still satisfies the condition in the definition of $A_{k, m}^{n}$

Note that by restricting the perturbed diffeomorphisms to $V$, the number of hyperbolic periodic points of period $\leq n$ is always $s$ Therefore, by repeating the 
construction at most $4 s$ times, we change all branches of $f$ with period $\leq n$, one at a time, into branches that satisfy the condition in the definition of $A_{k, m}^{n}$, and obtain a diffeomorphism $g \in V \cap A_{k m}^{n} \subset U \cap A_{k m}^{n}$ Hence $A_{k, m}^{n}$ is dense in $D_{\eta}^{r}(M)$ for all $n, k, m \in \mathbb{N}$

Let $B=\bigcap_{n k, m \in \mathbb{N}} A_{k, m}^{n} B$ is a residual subset of $D_{\eta}^{r}(M)$

Let $L$ be a branch of $f \in B$ Denote by $n$ the period of inf $L$, and take $k$ large enough so that $1 / k<$ length ( $L$ ) Since $f \in A_{k, m}^{n}$ for all $m \in \mathbb{N}$ and $L$ satisfies (1) and (2) in the definitions of the sets $A_{k, m}^{n}$ for $n$ and $k$ defined above, we have that $d\left[O_{+}\left(q_{k}(L), f\right), q_{k}(L)\right]<1 / m$ for all $m \in \mathbb{N}$, and hence $q_{k}(L)$ is a recurrent point, which proves the lemma

When $M$ is the sphere or the torus, it turns out that for the generic $f$ given by lemma 3, every hyperbolic periodic point has homoclinic points We conjecture that the same is true for compact surfaces of higher genus

Now we prove the following

LEMMA 5 Let $f$ be a $C^{1}$-diffeomorphism of a surface and $L_{1}, L_{2}, L_{3}$ branches of $f$ If $L_{3} \subset \omega\left(L_{2}\right)$ and $L_{2} \subset \omega\left(L_{1}\right)$, then $L_{3} \subset \omega\left(L_{1}\right)$

Proof Let $V$ be a neighbourhood of $x \in L_{3}$ and $\tilde{V}=V \backslash C$, where $C$ is the connected component of $L_{3} \cap V$ containıng $x$ It suffices to show that $L_{1} \cap \tilde{V} \neq \varnothing$ (See figure 27 )

Since $L_{3} \subset \omega\left(L_{2}\right)$, we have $L_{2} \cap \tilde{V} \neq \varnothing$ Take $y \in L_{2}$ and a neighbourhood $W$ of $y$ contained in $\tilde{V} L_{2} \subset \omega\left(L_{1}\right)$ implies that $L_{1} \cap W \neq \varnothing$, and therefore $L_{1} \cap \tilde{V} \neq \varnothing$

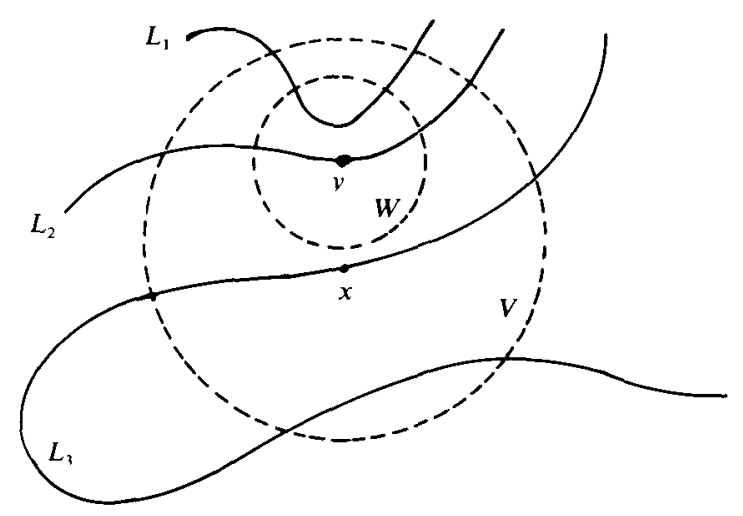

FiguRE 27

The last step before theorem 1 is the following

LEMMA 6 Let $f$ be a $C^{1}$ area-preserving diffeomorphism of a compact orientable surface and $L$ a branch of $f$ If $O(L) \cap \omega(L) \neq \varnothing$ then $L \subset \omega(L)$

Proof We have that $L=O\left(D, f^{\tau}\right)$, where $\tau$ is the period of $L$, and $D$ is a fundamental domain of $L$ Hence $f^{\prime} L \cap \omega(L) \neq \varnothing$ for some $t \in\{0, \quad, \tau-1\}$ 
Recall that $\omega(L)=\omega\left(D, f^{\tau}\right)$, and therefore

$$
f^{k} \omega(L)=f^{k} \omega\left(D, f^{\tau}\right)=\omega\left(f^{k} D, f^{\tau}\right)=\omega\left(f^{k} L\right) \quad \text { for any } k \in \mathbb{Z}
$$

We claim that $f^{n t} L \subset \omega(L)$, for all $n \geq 1$

By lemma 1, we have that $f^{\prime} L \subset \omega(L)$, and the claim is true for $n=1$ Assuming it to be true for $n$, we have that

$$
f^{(n+1)^{2}} L=f^{\prime}\left(f^{n} L\right) \subset f^{t}(\omega(L))=\omega\left(f^{t} L\right),
$$

but since $f^{\prime} L \subset \omega(L)$, lemma 5 implies that $f^{(n+1)} L \subset \omega(L)$, proving the claim

Since $L=f^{\pi t} L$, we have $L \subset \omega(L)$

From lemmas 3 and 6 we have the following

THEOREM 1 Let $M$ be a compact orientable surface and $1 \leq r \leq \infty$ There is a residual subset $B$ of $D_{\eta}^{r}(M)$ such that any branch $L$ of $f \in B$ satisfies $L \subset \omega(L)$

In fact, if $L$ is an unstable branch of period $\tau$ and $x \in L$ is $f$-recurrent, then for some $\imath \in\{0, \quad, \tau-1\}$, we have that $f^{\prime} x \in \omega\left(x, f^{\tau}\right)$, implying that $f^{\prime} L \cap \omega(L) \neq \varnothing$

\section{Pixton's theorem and its extension to the torus}

In this section we prove the following

THEOREM 2 Let $f$ be a $C^{1}$ area-preserving diffeomorphism of the sphere or the torus, and $p$ a hyperbolic periodic point of $f$ If each unstable (stable) branch of $p$ has a $f$-recurrent $\left(f^{-1}\right.$-recurrent) point, then $p$ has homoclinic points

From lemma 3 and theorem 2 it follows that for a $C^{r}$-generic $f$ in $D_{\eta}^{r}(M)$, every hyperbolic periodic point of $f$ has homoclinic points On the other hand, it is known that $C^{r}$-generically, all homoclinic points are transversal [6] Hence, we have the following

Theorem 3 (Pixton's theorem and its extension to the torus) Let $M$ be the sphere or the torus and $1 \leq r \leq \infty$ There is a residual subset $B$ of $D_{\eta}^{r}(M)$ such that any hyperbolic periodic point of $f \in B$ has transverse homoclinic points

Now we start with the proof of theorem 2

By lemma 6 and the observation following theorem 1, we have $L \subset \omega(L)$ for the four branches of $p$

It suffices to consider the case when $f$ fixes the branches of $p$ For simplicitiy of notation we assume that, in a neighbourhood of $p, f$ is linear of the form

$$
(x, y) \rightarrow\left(\lambda^{-1} x, \lambda y\right), \quad \text { with } 0<\lambda<1
$$

By a disk we mean any set homeomorphic to an open ball in $\mathbb{R}^{2}$

We denote the invariant manifolds and branches of $p$ by $W^{u}, W^{s}$ and $W_{+}^{u}, W_{-}^{u}$, $W_{+}^{s}, W_{-}^{s}$, respectively

For $n \geq 1$ and positive small $\delta$, we consider neighbourhoods of $p$,

$$
V=V(n)=\left\{(x, y),|x| \leq \delta,|y| \leq \delta \text { and }|x y| \leq \delta^{2} \lambda^{2 n}\right\},
$$

where $f$ is still linear 
The four sectors of $V$ are defined as the closure of each of the four components of $\{(x, y) \in V, x \neq 0$ and $y \neq 0\}$, and numbered counterclockwise as shown in figure 31

Let $S$ be a sector of $V$ The entrance of $S$ is the set En $(S)=\{(x, y) \in S, \lambda \delta<|y| \leq \delta\}$ and the exit of $S$ is the set $\operatorname{Ex}(S)=\{(x, y) \in S, \lambda \delta<|x| \leq \delta\}$ En $(S)$ has the property that if the orbit of a point ever intersects $S$ in the future, then the first time it does 1t, is by intersecting En $(S)$ Similarly, a point that ever intersects $S$ in the past, does it for the first time by intersecting $S$ at $\operatorname{Ex}(S)$

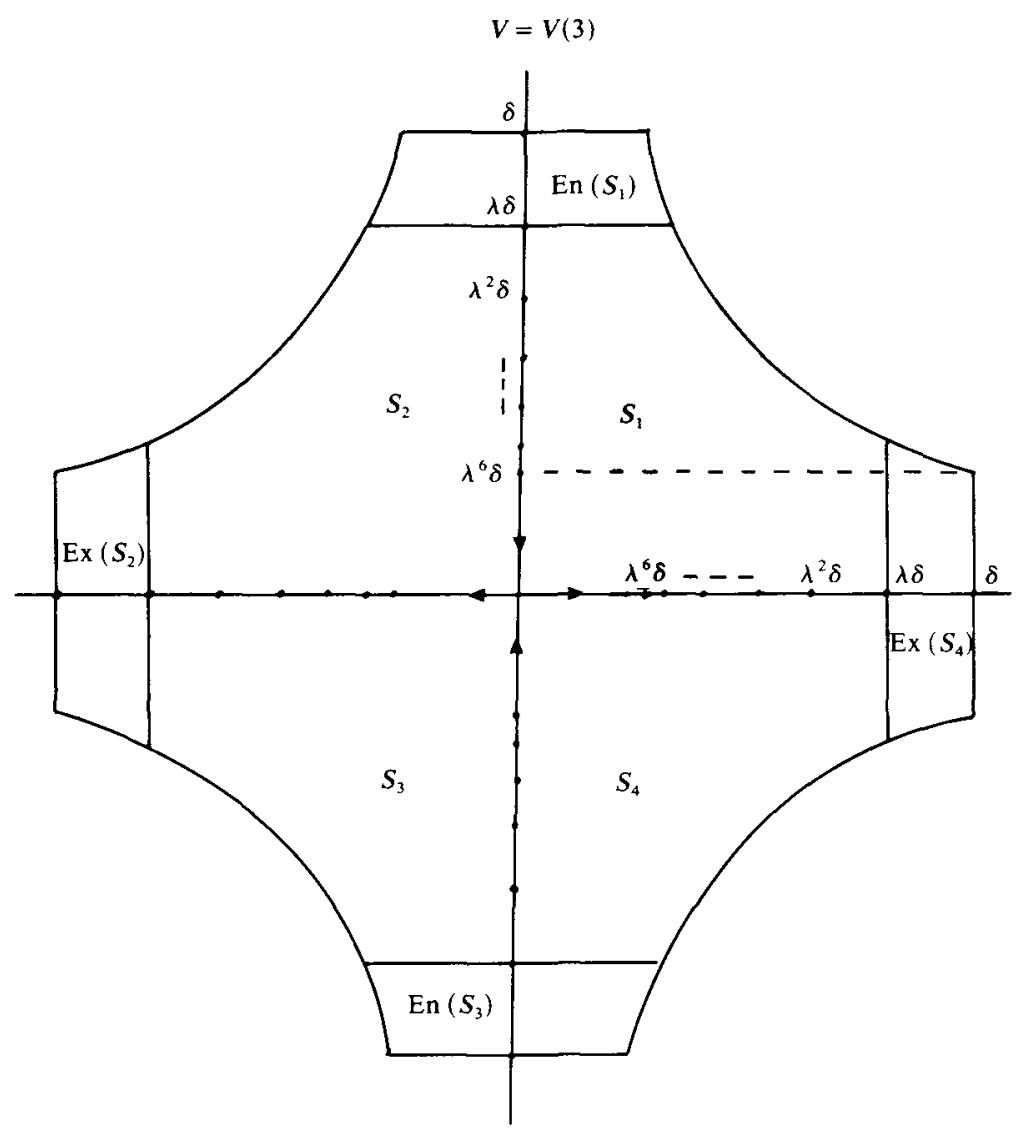

Figure 31

Let $W_{\text {loc }}^{u}=\{(x, y) \in V, y=0\}, W_{+ \text {loc }}^{u}=\{(x, y) \in V, y=0$ and $x>0\}$, and similarly define $W_{- \text {loc }}^{u}, W_{\text {loc }}^{s}, W_{\text {+loc }}^{s}$ and $W_{\text {-loc }}^{s}$

We speak of a simple curve as the embedded image of a circle or an interval

If $L$ is a branch of $p$ such that $L \backslash L_{\text {loc }}$ intersects $S$, then we define $q(L, S)$ as the first point of $L \backslash L_{\text {loc }}$ to intersect $S$ Let $\gamma(L, S)$ denote the gate of $L$ with respect to $S$, that is, $\gamma(L, S)$ is the straight open interval from $p$ to $q(L, S)$ 


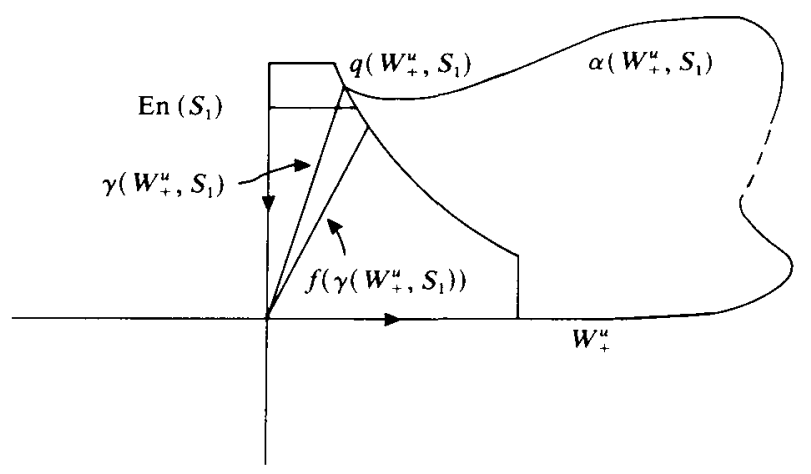

FIGURE 32

Let $\alpha(L, S)=L[\operatorname{mnf} L, q(L, S)] \cup \gamma(L, S)$ We have that $\alpha(L, S)$ is a simple closed curve If $L$ is an unstable branch of $p$, then $q(L, S) \in \operatorname{En}(S)$ and $f(\gamma(L, S)) \cap$ $\alpha(L, S)=\varnothing$ Similarly, if $L$ is stable, then $q(L, S) \in \operatorname{Ex}(S)$ and $f^{-1}(\gamma(L, S)) \cap$ $\alpha(L, S)=\varnothing$ (See figure 32 )

If $p \in \omega(L)$, we say that $L$ accumulates on a sector $S$ provided the interior of $S$ contains points of $L$ arbitrarily close to $p$

Note that if $L$ accumulates on $S_{1}$ and $L \cap\left(W_{+}^{u} \cup W_{+}^{s}\right)=\varnothing$, then it follows from lemma 1 that $W_{+}^{u} \cup W_{+}^{s} \subset \omega(L)$ A similar statement is valid for the other three sectors

Now, the proof of theorem 2, when $M$ is the sphere $S^{2}$, is a trivial task In fact, we show that each of the four branches of $p$ has homoclinic points

Fix $V=V(1)$, and let us consider, say, $W_{+}^{u}$ Since $W_{+}^{u} \subset \omega\left(W_{+}^{u}\right), W_{+}^{u}$ accumulates on $S_{1}$ or $S_{4}$, say $S_{1}$ We show that $W_{+}^{u} \cap W_{+}^{s} \neq \varnothing$

Suppose this is not true By the Jordan Curve Theorem, $\alpha\left(\omega_{+}^{u}, S_{1}\right)$ is a simple closed curve whose complement in $S^{2}$ consists of two regions, each homeomorphic to an open disk, and with $\alpha\left(W_{+}^{u}, S_{1}\right)$ as a common boundary Since $W_{+ \text {loc }}^{s} \cap$ $\alpha\left(W_{+}^{u}, S_{1}\right)=\varnothing, W_{+ \text {loc }}^{s} 1 \mathrm{~s}$ contained in one of the components of $S^{2} \backslash \alpha\left(W_{+}^{u}, S_{1}\right)$ Call this component $D$ We claim that $W_{+}^{s} \subset D$

If this is not the case, then $\left\{q \in W_{+}^{s}, q \notin D\right\}$ is not empty Let $q^{*}$ be its infimum Obviously $q^{*} \in \partial D=\alpha\left(W_{+}^{u}, S_{1}\right)$ and since $W_{+}^{u} \cap W_{+}^{s}=\varnothing$, we have that $q^{*} \in$ $\gamma\left(W_{+}^{u}, S_{1}\right)$, implying that $f\left(q^{*}\right) \in f\left(\gamma\left(W_{+}^{u}, S_{1}\right)\right) \subset S^{2} \backslash D$ (See figure 33(a)) On the other hand, $W_{+}^{s}$ is a stable branch, and hence $f\left(q^{*}\right)<q^{*}$ It follows from the definition of $q^{*}$ that $f\left(q^{*}\right) \in D$, a contradiction This proves the claim

Now, since $W_{+}^{s} \subset D$ and $\operatorname{Ex}\left(S_{1}\right) \subset S^{2} \backslash D$, we have that $W_{+}^{s}$ cannot accumulate on $S_{1}$ But $W_{+}^{s} \subset \omega\left(W_{+}^{s}\right)$, and therefore $W_{+}^{s}$ accumulates on $S_{2}$

We have that $W_{+}^{u}$ accumulates on $S_{1}$ and $W_{+}^{u} \cap W_{+}^{s}=\varnothing$ By lemma $1, W_{+}^{s} \subset \omega\left(W_{+}^{u}\right)$ and therefore $W_{+}^{u}$ accumulates on $S_{2}$ too

If we consider $\alpha\left(W_{+}^{u}, S_{2}\right)$ and argue as in the case of accumulation on $S_{1}$, we see that $\alpha\left(W_{+}^{u}, S_{2}\right)$ bounds a disk not containing $\operatorname{Ex}\left(S_{2}\right)$, and from which $W_{+}^{s}$ 
(a)

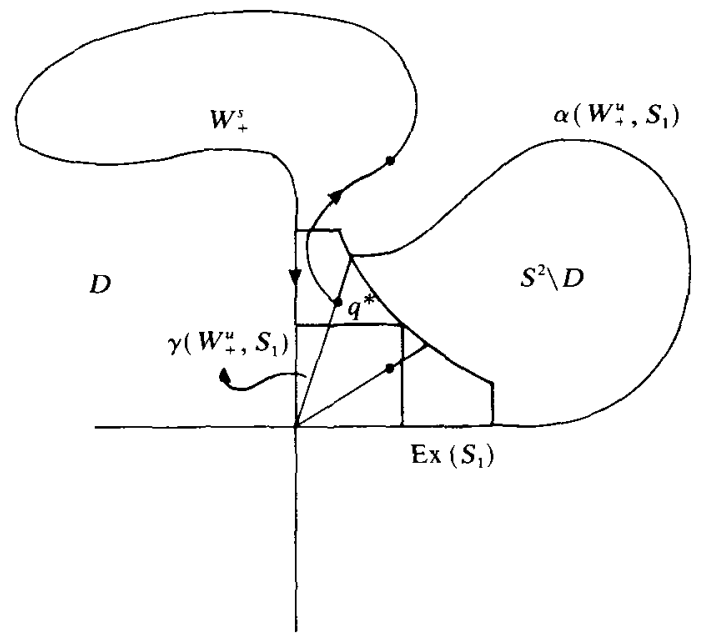

(b)

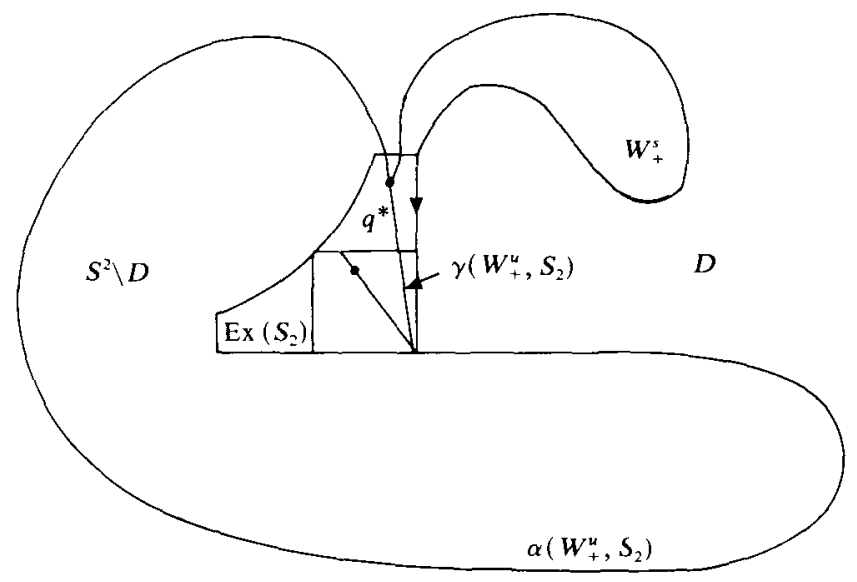

FiGuRE 33

cannot escape (See figure $33(\mathrm{~b})$ ) But this implies that $W_{+}^{s}$ cannot accumulate on $S_{2}$, a contradiction

This proves theorem 2 in the case $M=S^{2}$ Let us now do it when $M$ is the torus

Firstly we recall some basic facts about curves on surfaces, which can be found in chapter 6 of [9]

We regard the torus $T$ as the unit square, $[0,1] \times[0,1]$ with the sides identified in the usual way, and let $p$ be the point obtained by identifying the vertices of $[0,1] \times[0,1]$

Let $\pi \mathbb{R}^{2} \rightarrow T, \pi(x, y)=(x \bmod 1, y \bmod 1)$, be the usual covering map and $F$ be the lifting of $f$ to a diffeomorphism of $\mathbb{R}^{2}$ which fixes $0=(0,0)$ Note that $F$ leaves the form $\pi^{*} \eta$ invariant

For $q \in T$, the fibre over $q, \pi^{-1}(q)$, is just the orbit of $q$ under the group of isometries of the Euclidean plane $\mathbb{R}^{2}$ generated by translations by $(1,0)$ and $(0,1)$, 
where we regard $q$ as a point of $[0,1) \times[0,1) \subset \mathbb{R}^{2}$ This group of isometries is naturally isomorphic to $\mathbb{Z}^{2}$ and we simply write $\pi^{-1}(q)=\mathbb{Z}^{2}+q$ and call each of the points $r+q$ a translate of $q$

Denote the invariant manifolds and branches of 0 by $\hat{W}^{u}, \hat{W}^{s}$ and $\hat{W}_{+}^{u}, \hat{W}_{-}^{u}, \hat{W}_{+}^{s}$, $\hat{W}_{-}^{s}$, respectively A branch of 0 is the lifting of the corresponding branch of $p$

If $\alpha \subset T$ is a curve and $\hat{\alpha}$ is a lifting of $\alpha$, then $\alpha$ is simple if and only if $\hat{\alpha}$ is simple and the slices of $\pi^{-1}(\alpha)=\mathbb{Z}^{2}+\hat{\alpha}$ are pairwise disjoint (or intersect at most at their end points, when $\alpha$ is closed) Hence, if $\hat{L}$ is a branch of 0 , then $(r+\hat{L}) \cap$ $(s+\hat{L})=\varnothing$ if $r \neq s$, that is, the translates of a branch of 0 are pairwise disjoint

Let $\pi_{1}(T)$ be the fundamental group of $T$ based at $p$, and [ $\alpha$ ] be the equivalence class of a loop $\alpha$ at $p$ If we define $r(\alpha)$ as the endpoint of the liftıng of $\alpha$ starting at 0 , then $r(\alpha)$ depends only on $[\alpha]$, and $\phi \pi_{1}(T) \rightarrow \mathbb{Z}^{2}, \phi([\alpha])=r(\alpha)$, is a well defined isomorphism

If $f_{*}$ is the map induced by $f$ on $\pi_{1}(T)$ and $A=F / \mathbb{Z}^{2}$, then we have the following commutative diagram

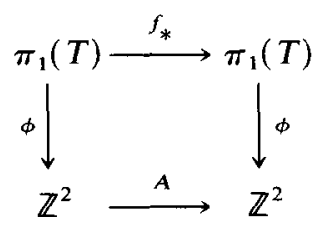

It is a fact that $A$ (or $f_{*}$ ) characterizes the homotopy class of $f$

For any $r \in \mathbb{Z}^{2}$ and $q \in \mathbb{R}^{2}$, we have $F(r+q)=F(r)+F(q)$ Therefore, if $\hat{L}$ is a stable branch of 0 , then

$$
r+\hat{L}=\left\{q \in \mathbb{R}^{2}, \lim _{n \rightarrow \infty} d\left(F^{n} q, F^{n} r\right)=0\right\},
$$

and if $\hat{L}$ is an unstable branch of 0 , then

$$
r+\hat{L}=\left\{q \in \mathbb{R}^{2}, \lim _{n \rightarrow \infty} d\left(F^{-n} q, F^{-n} r\right)=0\right\}
$$

In particular, if $r$ is a fixed point of $F$, then $r+\hat{L}$ is actually a branch of $r$

Consider the set $P=\left\{(m, n) \in \mathbb{Z}^{2}, \operatorname{gcd}(m, n)=1\right\}$ Let $\alpha$ be a loop at $p$ If $\alpha$ is simple, then $r(\alpha) \in P \cup\{0\}$, and conversely, if $r(\alpha) \in P \cup\{0\}$, then $\alpha$ is homotopic to a simple curve Furthermore, when $\alpha$ is simple, $T \backslash \alpha$ is disconnected if and only If $r(\alpha)=0$, and if $r(\alpha) \neq 0$, the infinite prolongation of $\alpha, \bigcup_{k \in \mathbb{Z}} k r(\alpha)+\hat{\alpha}$ is a simple curve that divides the plane into two regions

Now, let $\alpha$ and $\beta$ be two simple closed curves such that $\alpha \cap \beta=\{p\}$ Denote the intersection number of $\alpha$ and $\beta$ by $\#(\alpha, \beta)$ Then, the following are equivalent

(a) $\{r(\alpha), r(\beta)\}$ is linearly independent,

(b) $T \backslash(\alpha \cup \beta)$ is connected,

(c) $|\#(\alpha, \beta)|=1$,

(d) $|\operatorname{det}[r(\alpha), r(\beta)]|=1$

Denote the liftıngs of $\alpha$ and $\beta$ starting at 0 by $\hat{\alpha}$ and $\hat{\beta}$, respectıvely

If $r(\alpha)$ and $r(\beta)$ are linearly independent, then

$$
\hat{\alpha} \cup(r(\alpha)+\hat{\beta}) \cup \hat{\beta} \cup(r(\beta)+\hat{\alpha})
$$


1s a simple closed curve that bounds an open 'topological square' $Q$ with vertices $0, r(\alpha), r(\beta)$ and $r(\alpha)+r(\beta) \pi$ maps $Q$ diffeomorphically onto $T \backslash(\alpha \cup \beta)$, and $Q$ is a fundamental domain for the group of isometries of the Euclidean plane generated by translations by $(1,0)$ and $(0,1)$ We call $Q$ the square generated by $\alpha$ and $\beta$

We use $\hat{V}$ to denote a copy of $V$ centred at 0 , and write $\hat{S}, \widehat{\operatorname{En}}(S), \widehat{\mathrm{Ex}}(S), \hat{q}(L, S)$, $\hat{\gamma}(L, S)$ for the pullback under $\pi / \hat{V}$ of the corresponding subsets of $V$ If $L$ is a branch of $p$, let $\hat{\alpha}(L, S)$ be the liftıng of $\alpha(L, S)$ startıng at 0 , and $r(L, S)$ be the end point of $\hat{\alpha}(L, S)$ Note that the liftıng of $L[p, q(L, S)]$ starting at 0 is $\hat{L}[0, r(L, S)+\hat{q}(L, S)]$ and that

$$
\hat{\alpha}(L, S)=\hat{L}[0, r(L, S)+\hat{q}(L, S)] \cup(r(L, S)+\hat{\gamma}(L, S))
$$

(see figure 34 )

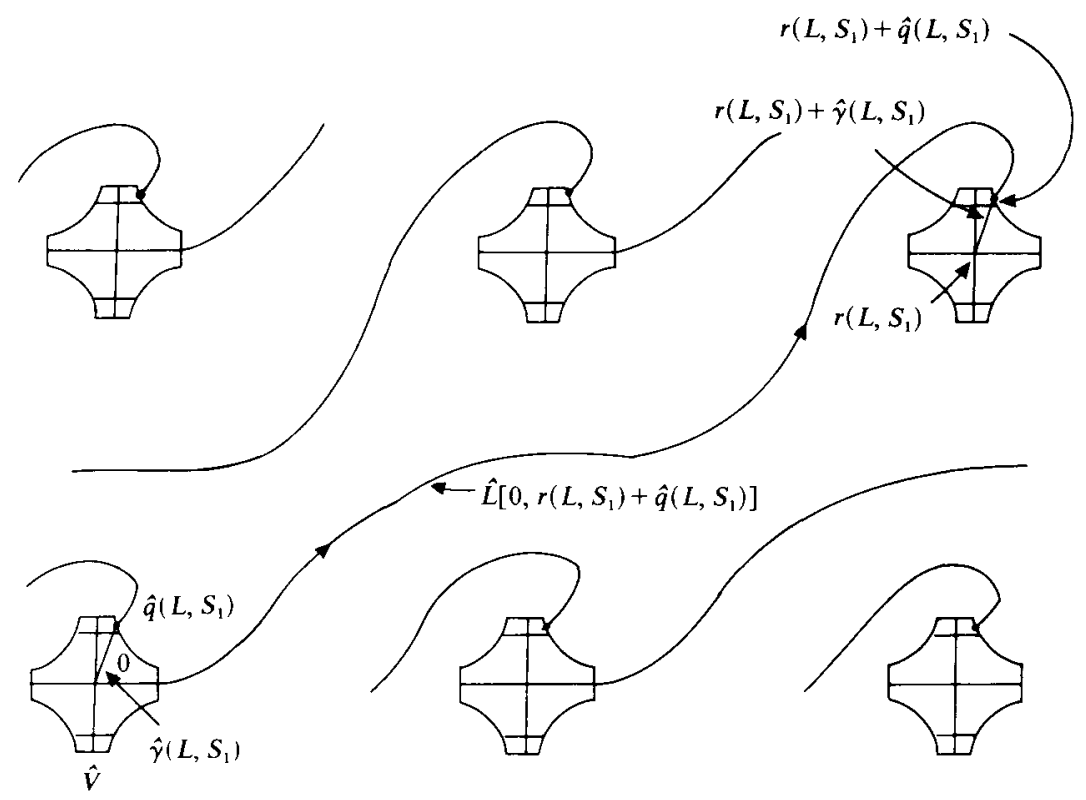

FIGURE 34

We say that $S_{1}$ and $S_{4}$ are the adjacent sectors of $W_{+}^{u}$, and that $W_{+}^{u}$ and $W_{+}^{s}$ are the adjacent branches of $S_{1}$ The same terminology will be used for the other branches and sectors

Observe that for $p$ to have a homoclinic point, it is enough that

$$
\left[\mathbb{Z}^{2}+\left(\hat{W}^{u} \backslash\{0\}\right)\right] \cap\left[\mathbb{Z}^{2}+\left(\hat{W}^{s} \backslash\{0\}\right)\right] \neq \varnothing
$$

Before starting with the next two lemmas, we make the convention that, if a branch $L$ of $p$ does not accumulate on a sector $S$, then $L \cap S=\varnothing$ (since we could just take the $\delta$ in the definition of $V$ smaller)

Recall that $L \subset \omega(L)$ for the four branches of $p$ 
LEMMA 7 Let $S$ be a sector where $W_{+}^{u}$ accumulates If $W_{+}^{u} \subset \overline{W^{s}}$ and $r\left(W_{+}^{u}, S\right)=0$, then $p$ has homoclinic points

A similar result holds for any other branch of $p$

Proof Assume by contradiction that $p$ has no homoclinic points

Since $r\left(W_{+}^{u}, S\right)=0$, we have that $\alpha\left(W_{+}^{u}, S\right)$ is a simple closed curve whose complement in $T$ has two components

If $S=S_{2}$ or $S=S_{3}$, say, $S=S_{2}$, then $W_{+}^{s}$ accumulates on $S_{2}$, since it accumulates on $S_{1}$ or $S_{2}$, and if it accumulates on $S_{1}$, then by lemma 1 , it accumulates all over $W_{+}^{u}$, which accumulates on $S_{2}$ (See figure $35(\mathrm{a})$ )

On the other hand, $W_{+ \text {loc }}^{s}$ and $f\left(\gamma\left(W_{+}^{u}, S_{2}\right)\right)$ are in different components of $T \backslash \alpha\left(W_{+}^{u}, S_{2}\right)$, implying that $W_{+}^{s}$ never leaves the component containıng $W_{+ \text {loc }}^{s}$ But since $W_{+}^{s}$ and $\operatorname{Ex}\left(S_{2}\right)$ are in different components, $W_{+}^{s}$ cannot accumulate on $S_{2}$, a contradiction

If $S=S_{1}$ or $S=S_{4}$, say $S=S_{1}$, then $W_{+}^{u} \subset \overline{W^{s}}$ implies that one of the stable branches of $p$ accumulates on $S_{1}$ (See figure $35(\mathrm{~b})$ )

(a)

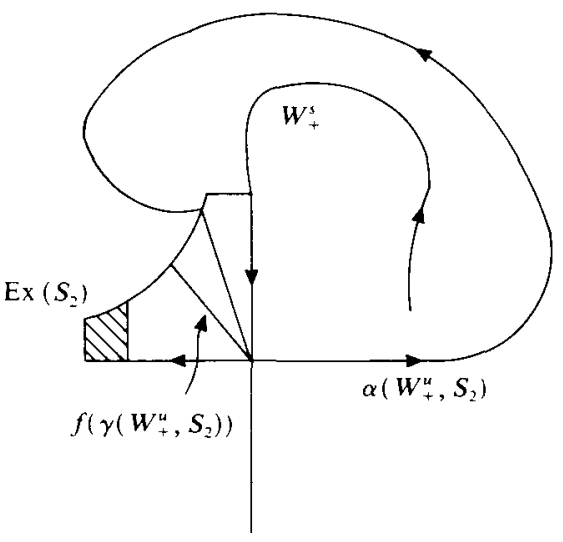

(b)

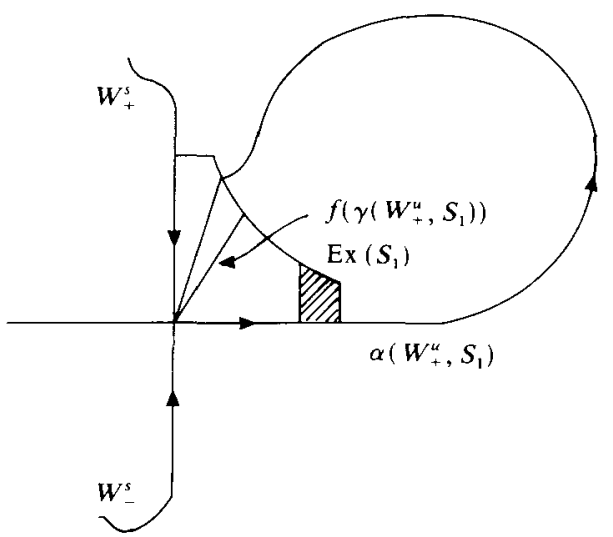

FIGURE 35 
On the other hand, $W_{\text {loc }}^{s}$ and $f\left(\gamma\left(W_{+}^{u}, S_{1}\right)\right)$ are in different components of $T \backslash \alpha\left(W_{+}^{u}, S_{1}\right)$, implying that $W^{s}$ never leaves the component containing $W_{\text {loc }}^{s}$ Since $W_{\text {loc }}^{s}$ and Ex $\left(S_{1}\right)$ are in different components, no stable branch of $p$ can accumulate on $S_{1}$, a contradiction

This proves lemma 7

LEMMA 8 If $f$ is homotopic to the identity and $p$ has no homoclinic points, then $\hat{W}_{+}^{u}$, $\hat{W}_{-}^{u}, \hat{W}_{+}^{s}$ and $\hat{W}_{-}^{s}$ are unbounded subsets of $\mathbb{R}^{2}$

Proof Firstly we show that if a branch of $p$ is contained in the closure of the other invariant manifold, then the corresponding branch of 0 is unbounded

Assume, say, $W_{+}^{u} \subset \overline{W^{s}}$ and let us show that $\hat{W}_{+}^{u}$ is unbounded

$W_{+}^{u}$ accumulates on $S_{1}$ or $S_{4}$, say on $S_{1}$ Since $W_{+}^{u} \subset \overline{W^{s}}$, by lemma 7 we have that $r\left(W_{+}^{u}, S_{1}, n\right) \neq 0$, for all $n \geq 1$ Note that $r$ depends on $n$ in the definition of $V=V(n)$

Assume by contradiction that $\hat{W}_{+}^{u}$ is bounded By lettıng $n \rightarrow \infty$, we have that the sequence $r\left(W_{+}^{u}, S_{1}, n\right)$ accumulates on some $r \in \mathbb{Z}^{2} \backslash\{0\}$, and that $\hat{W}_{+}^{u}$ accumulates on the sector $r+\hat{S}_{1}$ of $r+\hat{V}(1)$, for some $r \in \mathbb{Z}^{2} \backslash\{0\}$

Since $f$ is homotopic to the identity, every point of $\mathbb{Z}^{2}$ is fixed and $r+\hat{W}_{+}^{u}$ is actually an unstable branch of $r$ By lemma $2, \hat{W}_{+}^{u}$ accumulates all over $r+\hat{W}_{+}^{u}$

By the translation invariance of the branches of the points of $\mathbb{Z}^{2}$, we have that $r+\hat{W}_{+}^{u}$ accumulates all over $2 r+\hat{W}_{+}^{u}$ By lemma $5, \hat{W}_{+}^{u}$ accumulates all over $2 r+\hat{W}_{+}^{u}$

By induction, $\hat{W}_{+}^{u}$ accumulates all over $k r+\hat{W}_{+}^{u}$ for all $k \geq 1$, which contradicts the boundedness of $\hat{W}_{+}^{u}$

Now, let us prove the lemma Consider say $W_{+}^{u}$ It accumulates on $S_{1}$ or $S_{4}$, say $S_{1}$ By what we have just proved, $\hat{W}_{+}^{s}$ is unbounded

Assume by contradiction that $\hat{W}_{+}^{u}$ is bounded As above, $\hat{W}_{+}^{u}$ accumulates on a sector $r+\hat{S}_{1}$, for some $r \in \mathbb{Z}^{2}$ If $r \neq 0$, then the inductive argument given above implies that $\hat{W}_{+}^{u}$ accumulates all over $k r+\hat{W}_{+}^{u}$, for all $k \geq 1$, contradicting the boundedness of $\hat{W}_{+}^{u}$ If $r=0$, then $\hat{W}_{+}^{u}$ accumulates all over $\hat{W}_{+}^{s}$, which is unbounded by the first part of the proof Therefore $\hat{W}_{+}^{u}$ is also unbounded, again, a contradiction

Now we can prove theorem 2 when $M$ is the torus From now on, we assume by contradiction that $p$ has no homoclinic points

Firstly, we claim that there is a sector $S$ such that the two branches adjacent to $S$ accumulate on $S$ In fact, each branch accumulates on at least one adjacent sector Assume that $W_{+}^{u}$ accumulates on $S_{1}$ If the claim were false, then $W_{+}^{s}$ would accumulate on $S_{2}, W_{-}^{u}$ on $S_{3}$, and $W_{-}^{s}$ on $S_{4}$ But by the everywhere accumulation lemma, we would have $W_{-}^{u} \subset \omega\left(W_{+}^{s}\right), W_{-}^{s} \subset \omega\left(W_{-}^{u}\right)$ and $W_{+}^{u} \subset \omega\left(W_{-}^{s}\right)$, and by lemma 5 we would have $W_{+}^{u} \subset \omega\left(W_{+}^{s}\right)$, implying that $W_{+}^{s}$ also accumulates on $S_{1}$, a contradiction Hence we may assume that $W_{+}^{u}$ and $W_{+}^{s}$ accumulate on $S_{1}$

By lemma $7, r\left(W_{+}^{u}, S_{1}\right) \neq 0$ and $r\left(W_{+}^{s}, S_{1}\right) \neq 0$ Therefore $\alpha\left(W_{+}^{u}, S_{1}\right)$ and $\alpha\left(W_{+}^{s}, S_{1}\right)$ are simple closed curves that intersect exactly at $p$ with intersection number 1 or -1 (See figure 36 ) 


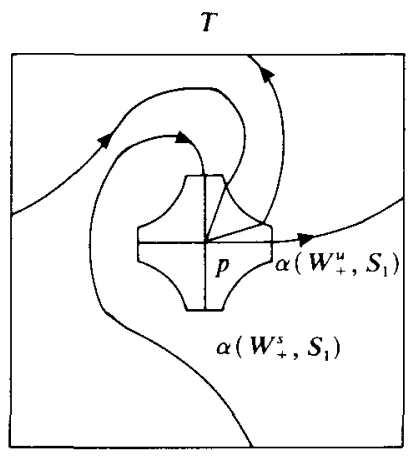

Figure 36

It follows that the infinite prolongations of $\alpha\left(W_{+}^{u}, S_{1}\right)$ and $\alpha\left(W_{+}^{s}, S_{1}\right)$, together with their translates, make a 'web' on $\mathbb{R}^{2}$, whose 'building blocks' are the translates of the square generated by $\alpha\left(W_{+}^{u}, S_{1}\right)$ and $\alpha\left(W_{+}^{s}, S_{1}\right)$ Call this square $Q$, and recall that $Q$ is the bounded component of

$$
\mathbb{R}^{2} \backslash\left[\hat{\alpha}\left(W_{+}^{u}, S_{1}\right) \cup \hat{\alpha}\left(W_{+}^{s}, S_{1}\right) \cup r\left(W_{+}^{s}, S_{1}\right)+\hat{\alpha}\left(W_{+}^{u}, S_{1}\right) \cup r\left(W_{+}^{u}, S_{1}\right)+\hat{\alpha}\left(W_{+}^{s}, S_{1}\right)\right]
$$

(See figure 37 )

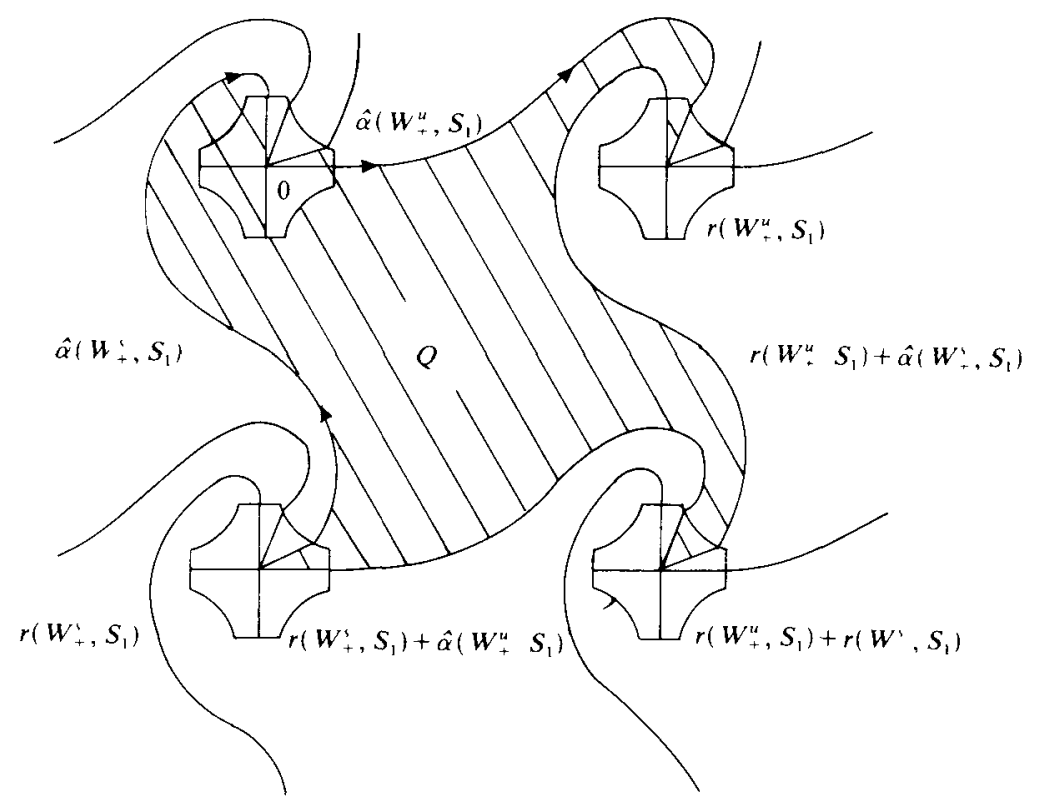

FIGURE 37

Let us consider now the case when $W_{-}^{u}$ or $W_{-}^{s}$ does not accumulate on $S_{1}$ It follows that $W_{-}^{u}$ and $W_{-}^{s}$ accumulate only on $S_{3}$ In fact, if say, $W_{-}^{u}$ does not accumulate on $S_{1}$, since $W_{+}^{u}$ and $W_{+}^{s}$ both do 1t, by lemma 1 we have that $W_{-}^{u}$ can 
not accumulate on $S_{2}$ nor on $S_{4}$ Hence $W_{-}^{u}$ accumulates only on $S_{3}$ Furthermore, by lemma 1 again, $W_{-}^{u}$ accumulates all over $W_{-}^{s}$, implying that $W_{-}^{s}$ accumulates only on $S_{3}$

Notice that $\hat{W}_{-10 c}^{u}$ and $\hat{W}_{- \text {loc }}^{s}$ are contained in $Q$, and that the gates in the boundary of $Q$ are all contained in $\mathbb{Z}^{2}+\hat{S}_{1}$ Since $W_{-}^{u}$ and $W_{-}^{s}$ never intersect $S_{1}$, we have that $\hat{W}_{-}^{u}$ and $\hat{W}_{-}^{s}$ are contained in $Q$ But $\left(\mathbb{Z}^{2}+\hat{S}_{3}\right) \cap Q=\hat{S}_{3}$, and therefore $r\left(W_{-}^{u}, S_{3}\right)=r\left(W_{-}^{s}, S_{3}\right)=0$ By lemma $7, p$ has homoclinıc points, a contradiction

Consider now the case when $W_{-}^{u}$ and $W_{-}^{s}$ accumulate on $S_{1}$, and therefore the four branches accumulate on $S_{1}$

From now on, we consider accumulations only on $S_{1}$, and simplify the notation as follows $\alpha_{+}^{u}=\alpha\left(W_{+}^{u}, S_{1}\right), \gamma_{+}^{u}=\gamma\left(W_{+}^{u}, S_{1}\right), q_{+}^{u}=q\left(W_{+}^{u}, S_{1}\right), r_{+}^{u}=r\left(W_{+}^{u}, S_{1}\right)$ and similarly for the other three branches of $p$ and the branches of 0

We have that $Q \cap\left(r+\widehat{\operatorname{En}}\left(S_{1}\right)\right) \neq \varnothing$ only for $r=r_{+}^{u}$ or $r=r_{+}^{u}+r_{+}^{s}$, and also that $Q \cap\left(r+\widehat{\operatorname{Ex}}\left(S_{1}\right)\right) \neq \varnothing$ only for $r=r_{+}^{s}$ or $r=r_{+}^{u}+r_{+}^{s}$ (See figure 37 ) Therefore $r_{-}^{u} \epsilon$ $\left\{r_{+}^{u}, r_{+}^{u}+r_{+}^{s}\right\}$ and $r_{-}^{s} \in\left\{r_{+}^{s}, r_{+}^{u}+r_{+}^{s}\right\}$

If we had $r_{-}^{u}=r_{+}^{u}$ or $r_{-}^{s}=r_{+}^{s}$, then $\hat{\alpha}_{-}^{u}$ and $\hat{\alpha}_{-}^{s}$ would intersect at a point not in $\mathbb{Z}^{2}$ (see figure 38 ), contradicting the fact that $\alpha_{-}^{u} \cap \alpha_{-}^{s}=\{p\}$

Hence $r_{-}^{u}=r_{-}^{s}=r_{+}^{u}+r_{+}^{s}$

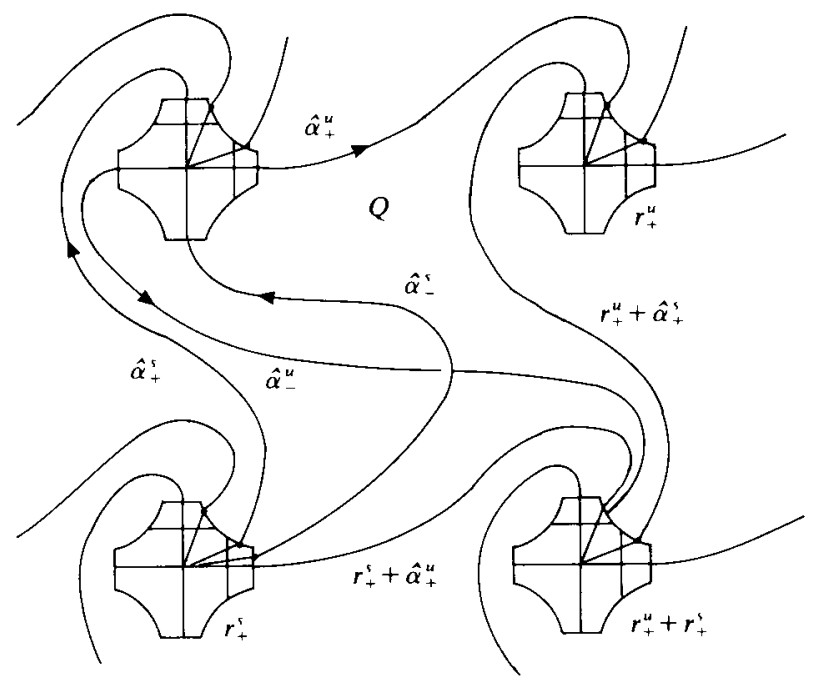

FIGURE 38

Let us now show that $f$ is homotopic to the identity For this, it is enough to show that $r_{-}^{u}$ and $r_{+}^{u}$ are fixed points of $F$

If $n$ in the definition of $V(n)$ is greater than 1 , then $f\left(\operatorname{En}\left(S_{1}\right)\right) \cap\left(\alpha_{+}^{s} \cup \alpha_{-}^{s}\right)=\varnothing$ (See figure 39) This implies that $f\left(\alpha_{-}^{u}\right) \cap\left(\alpha_{+}^{s} \cup \alpha_{-}^{s}\right)=\{p\}$, since $f\left(\alpha_{-}^{u}\right)=$ $W_{-}^{u}\left[p, f\left(q_{-}^{u}\right)\right] \cup f\left(\gamma_{-}^{u}\right)$ and $f\left(q_{-}^{u}\right) \in f\left(\operatorname{En}\left(S_{1}\right)\right)$ is the first point of $W_{-}^{u}$ to intersect $S_{1} \backslash \operatorname{En}\left(S_{1}\right)$ 


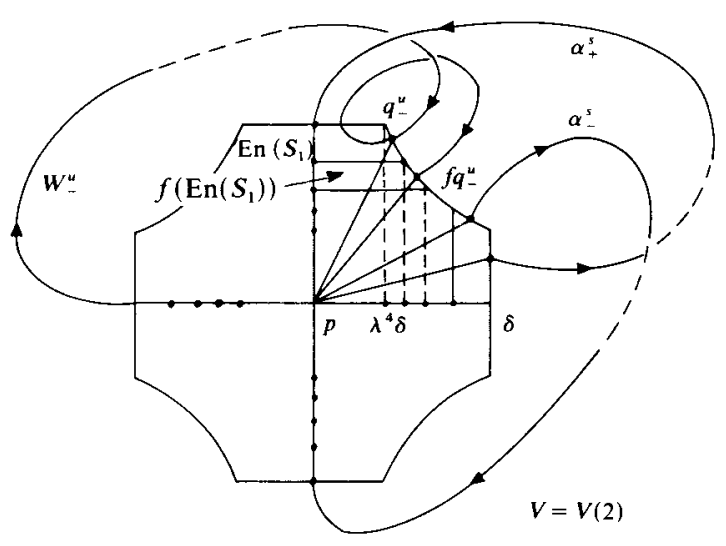

FIGURE 39

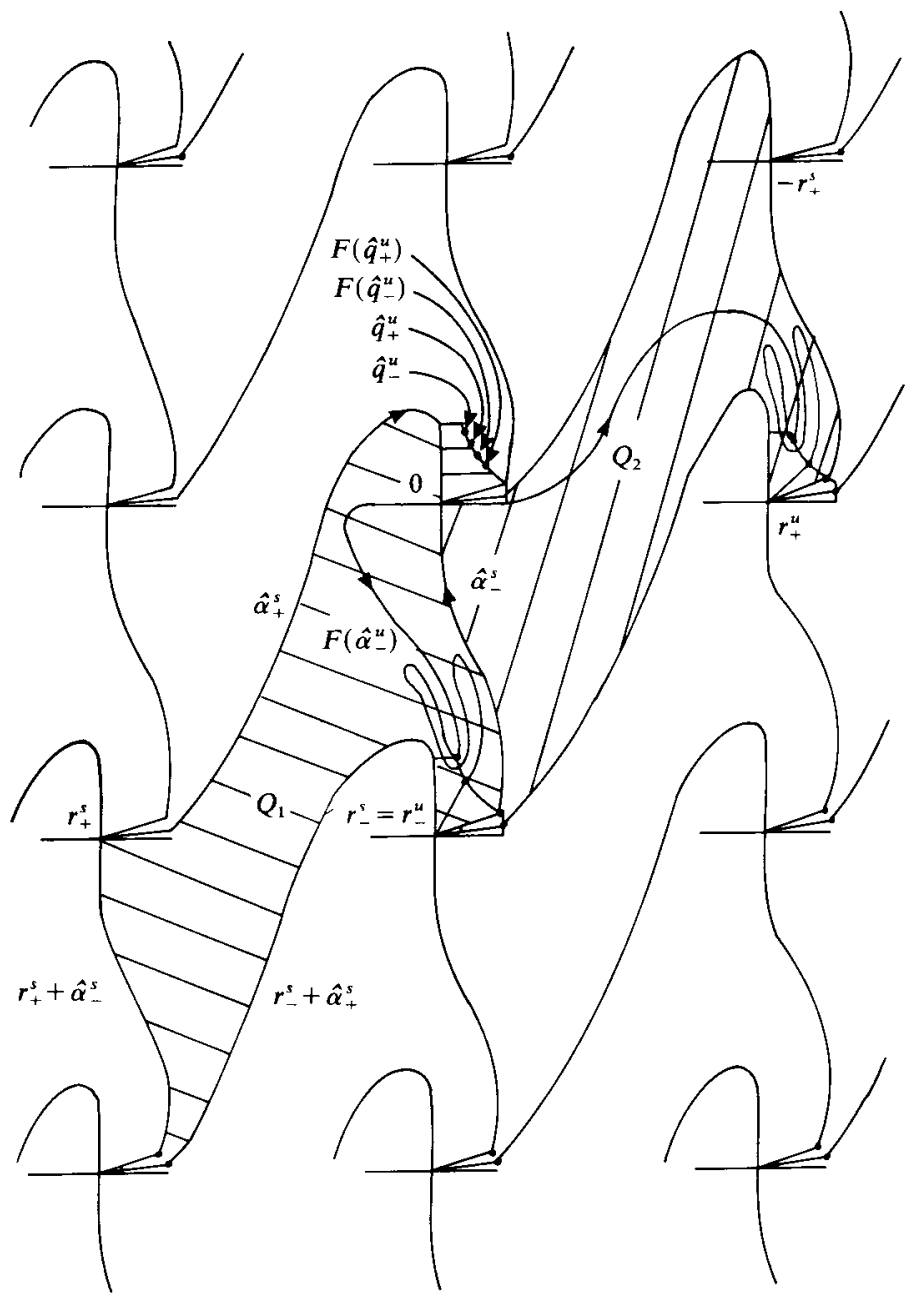

FIGURE 310 
Let $Q_{1}$ be the square generated by $\alpha_{+}^{s}$ and $\alpha_{-}^{s}$, that 1s, $Q_{1}$ is the bounded component of $\mathbb{R}^{2} \backslash\left[\hat{\alpha}_{+}^{s} \cup \hat{\alpha}_{-}^{s} \cup\left(r_{+}^{s}+\hat{\alpha}_{-}^{s}\right) \cup\left(r_{-}^{s}+\hat{\alpha}_{+}^{s}\right)\right]$ Note that $\hat{\alpha}_{-}^{u} \backslash\left\{0, r_{-}^{u}\right\}=\hat{W}_{-}^{u}\left(0, r_{-}^{u}+\hat{q}_{-}^{u}\right) \cup$ $\left(r_{-}^{u}+\hat{\gamma}_{-}^{u}\right)$ is contained in $Q_{1}$ (See figure 310 )

Since $f\left(\alpha_{-}^{u}\right) \cap\left(\alpha_{+}^{s} \cup \alpha_{-}^{s}\right)=\{p\}$, we have that

$$
F\left(\hat{\alpha}_{-}^{u} \backslash\left\{0, r_{-}^{u}\right\}\right)=\hat{W}_{-}^{u}\left(0, F\left(r_{-}^{u}+\hat{q}_{-}^{u}\right)\right) \cup F\left(r_{-}^{u}+\hat{\gamma}_{-}^{u}\right)
$$

cannot intersect the boundary of $Q_{1}$ Hence $\hat{W}_{-}^{u}\left(0, F\left(r_{-}^{u}+\hat{q}_{-}^{u}\right)\right) \subset Q_{1}$

On the other hand, we have that $\mathbb{Z}^{2}+\left\{F\left(\hat{q}_{-}^{u}\right)\right\} \cap Q_{1}=\left\{r_{-}^{u}+F\left(\hat{q}_{-}^{u}\right)\right\}$, and therefore $F\left(r_{-}^{u}+\hat{q}_{-}^{u}\right)=r_{-}^{u}+F\left(\hat{q}_{-}^{u}\right)$ But since $F\left(r_{-}^{u}\right)+F\left(\hat{q}_{-}^{u}\right)=F\left(r_{-}^{u}+\hat{q}_{-}^{u}\right)$, we have $F\left(r_{-}^{u}\right)=r_{-}^{u}$

If we consider the square $Q_{2}=-r_{+}^{s}+Q_{1}$, which contains $\hat{\alpha}_{+}^{u} \backslash\left\{0, r_{+}^{u}\right\}$, then the same argument works to show that $F\left(r_{+}^{u}\right)=r_{+}^{u}$ (See figure 310 )

Now that $f$ is homotopic to the identity, lemma 8 implies that the four branches of 0 are unbounded

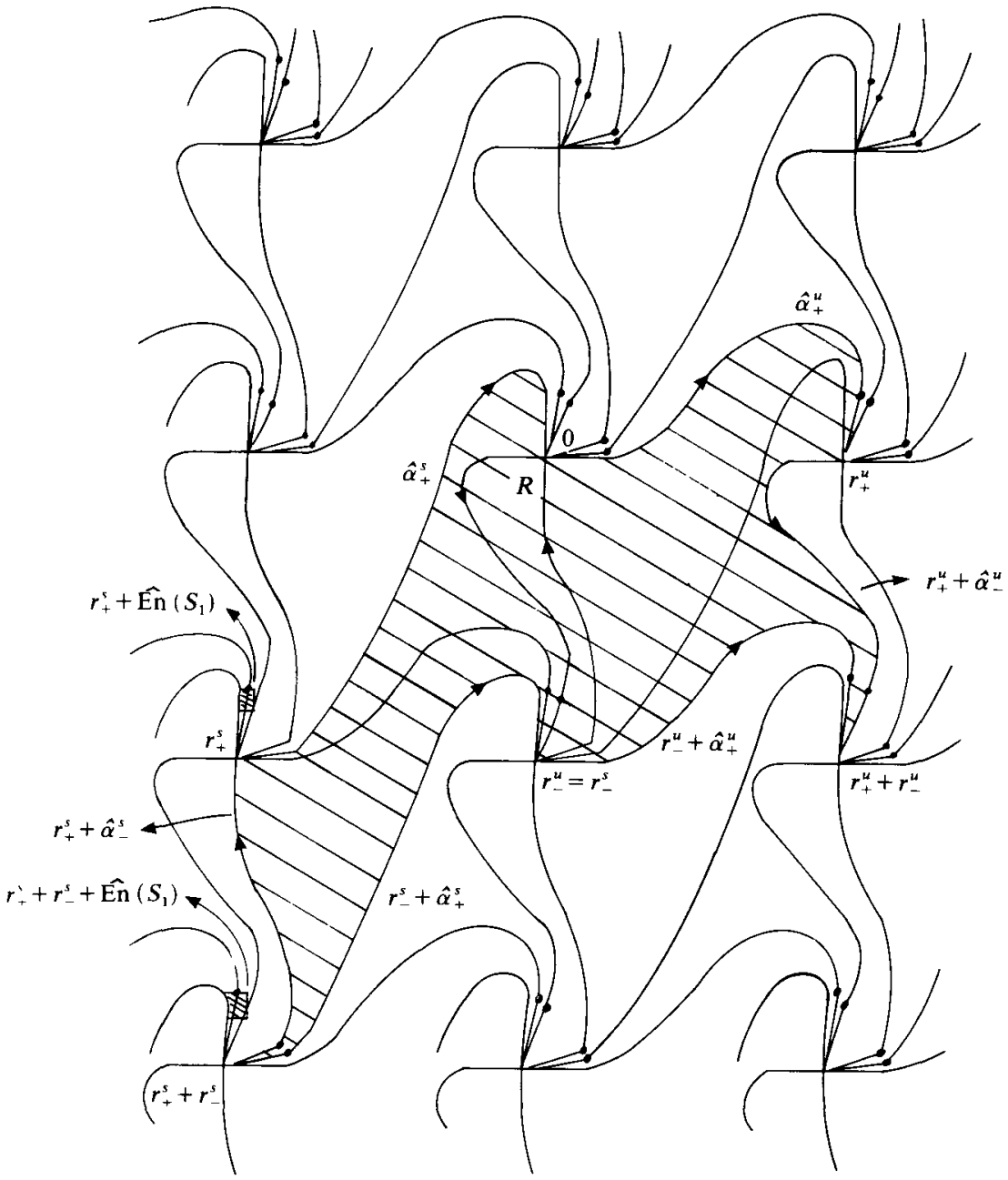

FIgURE 311 
Observe that every point in $\mathbb{Z}^{2}$ is fixed by $F$ and that the translates of the branches of 0 are also branches (invariant sets)

Let $R$ be the union of the squares generated by $\alpha_{+}^{u}, \alpha_{-}^{u}$ and by $\alpha_{+}^{s}, \alpha_{-}^{s}$ The boundary of $R$ is the simple closed curve

$$
\hat{\alpha}_{+}^{u} \cup\left(r_{+}^{u}+\hat{\alpha}_{-}^{u}\right) \cup\left(r_{-}^{u}+\hat{\alpha}_{+}^{u}\right) \cup\left(r_{-}^{s}+\hat{\alpha}_{+}^{s}\right)+\left(r_{+}^{s}+\hat{\alpha}_{-}^{s}\right) \cup \hat{\alpha}_{+}^{s}
$$

(See figure 311 )

Consider $\hat{W}_{-}^{u} \hat{W}_{- \text {loc }}^{u} \subset R$, and by its unboundedness, $\hat{W}_{-}^{u}$ must leave $R$

Look at the six gates in the boundary of $R$ Since there are no homoclinic points, $\hat{W}_{-}^{u}$ must leave $R$ by intersecting one of the gates Let $q^{*}$ be the first point of $\hat{W}_{-}^{u}$ to intersect the gates in the boundary of $R$

Since $r_{+}^{s}+\widehat{\operatorname{En}}\left(S_{1}\right)$ and $r_{+}^{s}+r_{-}^{s}+\widehat{\operatorname{En}}\left(S_{1}\right)$ are outside of $R, q^{*}$ must belong to one of the three unstable gates, $r_{+}^{u}+\hat{\gamma}_{+}^{u}, r_{+}^{u}+r_{-}^{u}+\hat{\gamma}_{+}^{u}$ and $r_{+}^{u}+r_{-}^{u}+\hat{\gamma}_{-}^{u}$

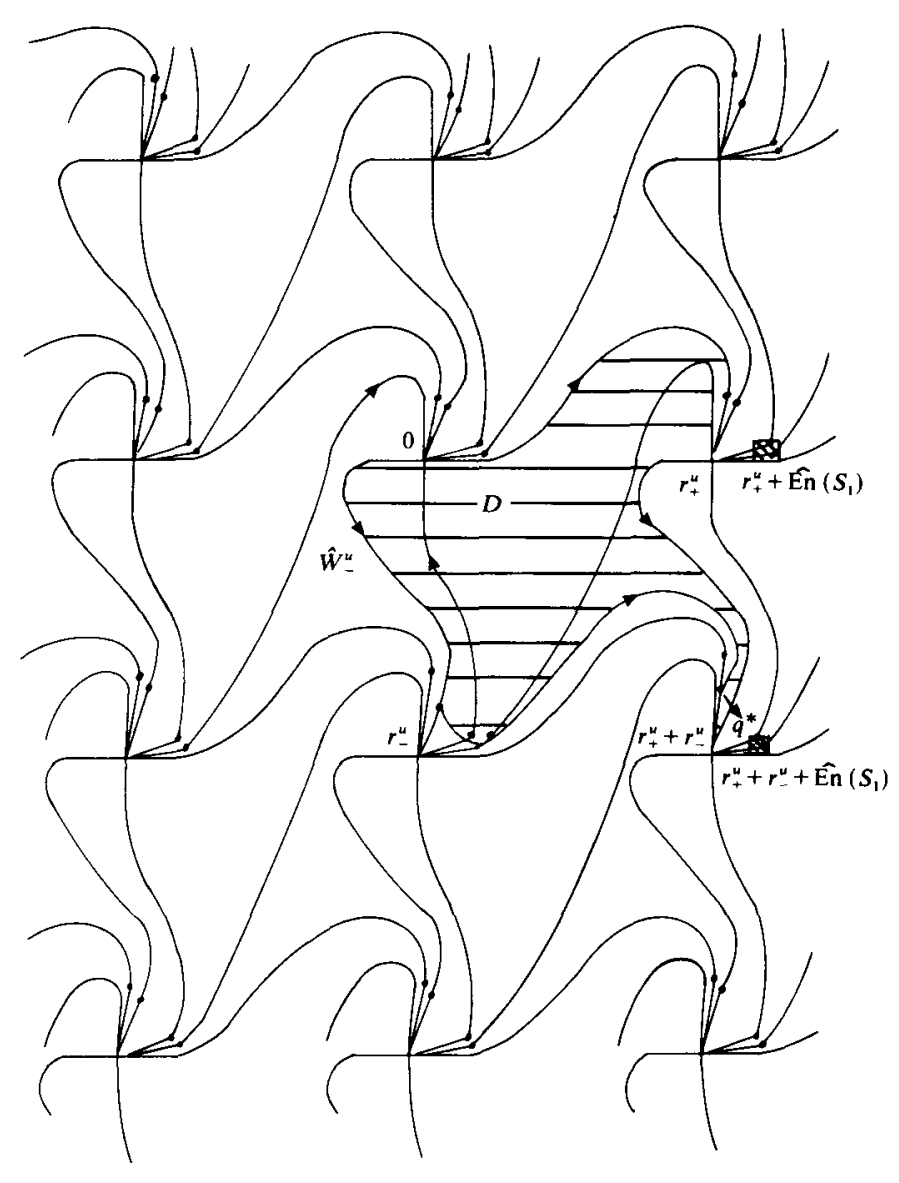

FIGURE 312 
Consider the case when $q^{*} \in r_{+}^{u}+r_{-}^{u}+\hat{\gamma}_{+}^{u}$ Let $I$ be the subinterval of $r_{+}^{u}+r_{-}^{u}+\hat{\gamma}_{+}^{u}$ from $r_{+}^{u}+r_{-}^{u}$ to $q^{*}$, and consider the simple closed curve

$$
\beta=\hat{W}_{-}^{u}\left[0, q^{*}\right] \cup I \cup\left(r_{+}^{u}+\hat{\alpha}_{-}^{u}\right) \cup \hat{\alpha}_{+}^{u}
$$

(See figure 312 ) If we denote by $D$ the bounded component of $\mathbb{R}^{2} \backslash \beta$, then $\hat{W}_{- \text {loc }}^{s} \subset D$ On the other hand, $r_{+}^{u}+\widehat{\operatorname{Ex}}\left(S_{1}\right)$ and $r_{+}^{u}+r_{-}^{u}+\widehat{\mathrm{Ex}}\left(S_{1}\right)$ are outside of $R$, 1mplying that $\hat{W}_{-}^{s}$ cannot leave $D$ But this is a contradiction since $\hat{W}_{-}^{s}$ is unbounded and $D$ is not In the other two cases we make similar constructions If $q^{*} \in r_{+}^{u}+\hat{\gamma}_{+}^{u}$, take $\beta$ as $\hat{W}_{-}^{u}\left[0, q^{*}\right] \cup \hat{\alpha}_{+}^{u}\left[0, q^{*}\right]$

and if $q^{*} \in r_{+}^{u}+r_{-}^{u}+\hat{\gamma}_{-}^{u}$, take $\beta$ as

$$
\hat{W}_{-}^{u}\left[0, q^{*}\right] \cup \hat{\alpha}_{+}^{u} \cup\left(r_{+}^{u}+\hat{\alpha}_{-}^{u}\right)\left[r_{+}^{u}, q^{*}\right]
$$

(We are using the subinterval notation for $\hat{\alpha}_{+}^{u}$ and $r_{+}^{u}+\hat{\alpha}_{-}^{u}$ See figures 313 and

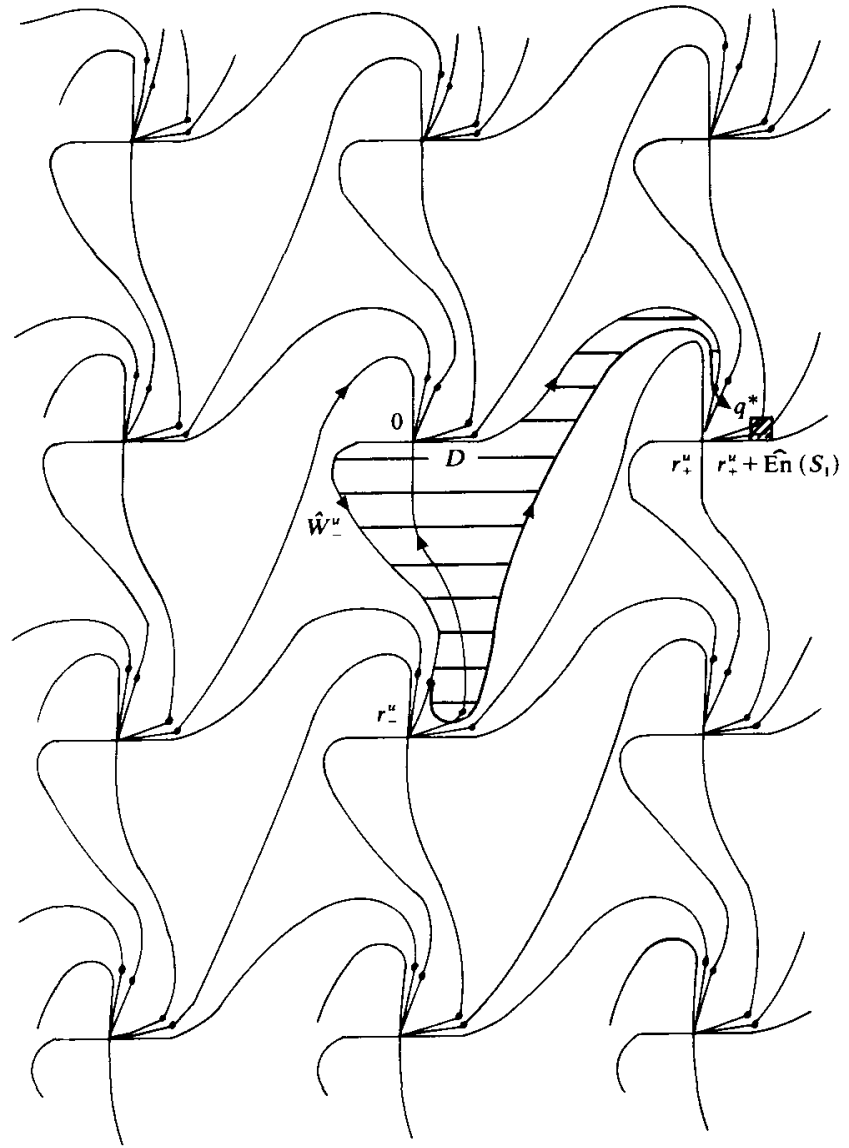

FIGURE 313 


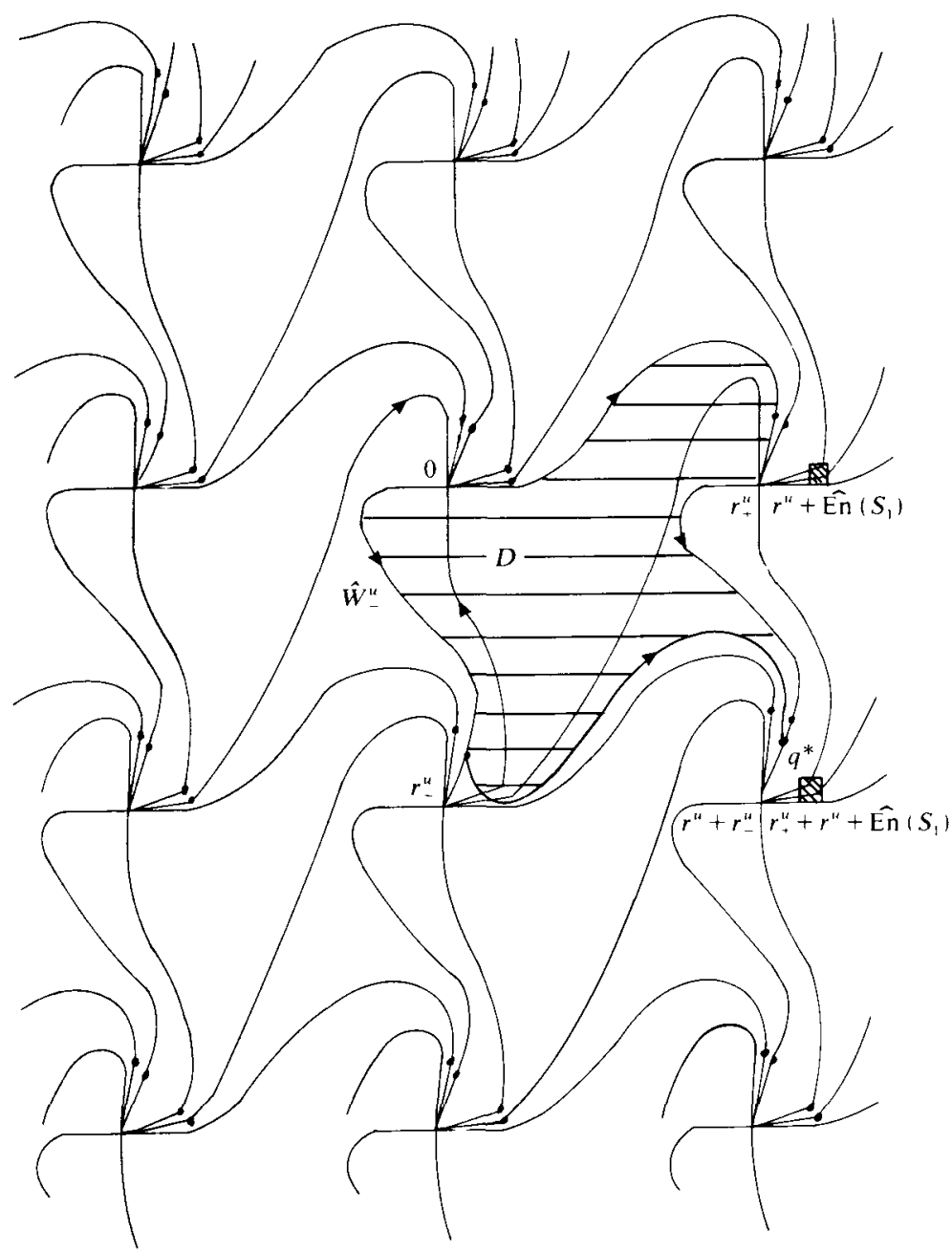

FIGURE 314

314 ) As before, if $D$ is the bounded component of $\mathbb{R}^{2} \backslash \beta$, then $D$ contains $\hat{W}_{- \text {loc }}^{s}$ and $r_{+}^{u}+\widehat{\mathrm{EX}}\left(S_{1}\right)$ and $r_{+}^{u}+r_{-}^{u}+\widehat{\mathrm{EX}}\left(S_{1}\right)$ are outside of $D$ This implies that $\hat{W}_{-}^{s}$ never leaves $D$, a contradiction

Th1s ends the proof of theorem 2

Finally, we would like to make a few remarks

There is a more direct argument for theorem 1, that works when $M$ is any compact manıfold (of any dimension), and we consider diffeomorphisms of $M$ that preserve a measure with smooth density In lemma 3, we show that for generic $f$, given a hyperbolic periodic point of $f$, its unstable (stable) invariant manifold contains a $f$-recurrent $\left(f^{-1}\right.$-recurrent) point With a slight change in the argument of the mentioned lemma, we can show that for a generic $f$, given a hyperbolic periodic point of $f$, its unstable (stable) invariant manifold has a fundamental domain with 
a countable dense subset of $f$-recurrent $\left(f^{-1}\right.$-recurrent) points On the other hand, an inductive argument, similar to that in the proof of lemma 6 , shows that a $f$-recurrent point is also $f^{n}$-recurrent for any $n>1$ Therefore, for a generic $f$, given a hyperbolic periodic point of $f$ with period $\tau$, its unstable (stable) invariant manifold has a fundamental domain with a countable dense subset of $f^{\tau}$-recurrent ( $f^{-\tau}$-recurrent) points It follows that the invariant manifolds accumulate all over themselves

Hence, if $M$ is a compact manifold, $D_{\eta}^{r}(M)$ is the space of all $C^{r}$-diffeomorphisms of $M$ that preserve a smooth invariant measure $\eta$, topologized with the $C^{r}$-topology, $1 \leq r \leq \infty$, then $D_{\eta}^{r}(M)$ has a residual subset $B$ such that if $p$ is a hyperbolic periodic point of $f \in B$, then each of the invariant manifolds of $p$ is contained in its omega-limit set

Theorem 3 is also true for the Projective Plane and the Klein Bottle This follows immediately from the above remark, theorem 2 and a two to one cover In particular, to prove theorem 3 for an arbitrary compact surface, it suffices to prove theorem 2 in the orientable case Our feeling is that theorem 2 is true for an arbitrary compact surface, and a proof following the lines of this work would involve an understanding of the accumulation of a branch over its translates in the universal cover

\section{REFERENCES}

[1] G D Bırkhoff Collected Mathematıcal Papers Vol II, p 350, American Mathematical Society, 1950

[2] J N Mather Invariant subsets for area-preserving homeomorphisms of surfaces, mathematical analysis and applications, Part B Advances in Mathematics Supplementary Studies, Volume 7B

[3] S E Newhouse Quası-elliptıc periodıc points in conservative dynamical systems Amer $J$ Math Vol 99 (1977) No 5, 1061-1087

[4] D Pixton Planar homoclinic points J Differential Equations 44 (1982), 365-382

[5] H Poincare Les Methodes Nouvelles de la Mécantque Celeste, Tome II 1899

[6] C Robinson Generic Properties of conservative systems, I, II, Amer J of Math 92 (1970), 562-603, 897-906

[7] C Robinson Closing stable and unstable manifolds on the two-sphere Proc Amer Math Soc 41 (1973), 299-303

[8] S Smale Diffeomorphisms with many periodic points In Differential and Combinatorial Topology, Princeton University Press, Prunceton, New Jersey, 1965

[9] J Stıllwell Classical Topology and Combınatorial Group Theory Graduate Texts in Mathematics, Volume 72, Springer-Verlag

[10] F Takens Homoclinic points in conservatıve systems Invent Math 18 (1972), 267-292

[11] E Zehnder Homoclinic points near elliptic fixed points Comm Pure Appl Math XXVI (1973), 131-182 